\title{
Medizinische Apps in der HNO-Heilkunde Medical Apps in Oto-Rhino-Laryngology
}

(c) (1) (): $\odot$

\author{
Autoren \\ Kristen Rak ${ }^{1}$, Johannes Völker¹, Johannes Taeger ${ }^{1}$, Andreas Bahmer ${ }^{1}$, Rudolf Hagen ${ }^{1}$, Urs-Vito Albrecht ${ }^{2}$
}

Institute

1 Klinik und Poliklinik für Hals-, Nasen- und Ohrenkrankheiten, plastische und ästhetische Operationen, Universitätsklinikum Würzburg

2 Peter L. Reichertz, Institut für Medizinische Informatik, Medizinische Hochschule Hannover

Schlüsselwörter

Apps, Chancen, Risiken, Literaturrecherche, Umfrage, App-Entwicklung

Bibliografie

DOI https://doi.org/10.1055/a-0740-4866

Laryngo-Rhino-Otol 2019; 98: S253-S272

(c) Georg Thieme Verlag KG Stuttgart · New York

ISSN 0935-8943

Korrespondenzadresse

Priv.-Doz. Dr. med. Kristen Rak

Univ. HNO-Klinik

Josef-Schneider-Straße 11

D-97080 Würzburg

rak_k@ukw.de

\section{ZUSAMMENFASSUNG}

Die Nutzung mobiler Informations- und Kommunikationstechnologie im Kontext medizinscher und gesundheitsnaher Dienstleistungen, z. B. in Form von Apps, gewinnt, leider jedoch häufig unter Missachtung notwendiger Qualitätskriterien, immer mehr an Bedeutung. So erscheint es wichtig, dass neben einer die Anwendungssoftware kontrollierenden Instanz möglichst hoch qualifizierte Akteure des Gesundheitswesens bei der Erstellung mitwirken. Schon aus Haftungsgründen muss der beratende Arzt eine hohe Sorgfalt bei der Auswahl und Empfehlung einer App walten lassen, gerade auch in Anbetracht der Tatsache, dass nur wenige Apps als Medizinprodukt zertifiziert sind. Auf dem Markt gibt es eine große Anzahl an medizinischen Apps, wobei nur ein geringer Anteil dieser auf den Fachbereich HNO-Heilkunde entfällt. Prozentual sind die Teilbereiche Audiologie, Schlafmedizin und Allergologie am häufigsten vertreten. Obwohl sich der Fachbereich der HNO-Heilkunde zunehmend mit dieser Thematik wissenschaftlich auseinandersetzt, fehlt, wie generell bei vielen medizinischen Apps, eine wissenschaftliche Evidenz der Inhalte und Ergebnisse. Es gibt jedoch für Anwender weitere Möglichkeiten, medizinische Apps nach definierten Qualitätskriterien in verschiedenen Kategorien, wie z. B. Funktionalität, Wissenschaftlichkeit, aber auch Datenschutz, zu beurteilen. Keine der mittels eines solchen Bewertungstools evaluierten Apps erfüllte alle geforderten Kriterien, dem Anwender steht jedoch hiermit ein Instrument zur besseren Einschätzung der Anwendungssoftware zur Verfügung. Im Rahmen des Entwicklungsprozesses einer medizinischen App für den HNO-Bereich war es jedoch möglich, die
Qualitätskriterien zu berücksichtigen. Zusammenfassend soll die vorliegende Arbeit einen Überblick über Thema „Apps in der HNO-Heilkunde“ ermöglichen mit dem Ziel, dieses moderne Hilfsmittel nutzbringend einsetzen zu können.

\section{ABSTRACT}

The implementation of mobile information and communication technology in the field of health services, e. g. in the form of apps, is becoming increasingly important. Unfortunately, the necessary quality criteria are often mising. Thus, it seems important, that in addition to an app controlling authority highly qualified health care professionals participate in the development of these applications. For reasons of liability, however, the physician must exercise great caution in the selection and recommendation of medical apps, especially considering, that only a few apps are certified as medical devices. There are a large number of medical apps on the market, with only a small proportion being assigned to the field of otorhinolaryngology. The areas of audiology, sleep medicine and allergology are most frequently represented. Althouhgh there is increasing scientific work on this topic in the field of otorhinolaryngology, there is a lack of scientific evidence of contents and results, as is generally the case of medical apps. However, there are other possibilities for users to rate medical apps regarding defined qualitiy criteria such as functionality, scientific integrity, but also data privacy. None of the apps assessed by such a evaluation tool met all the required quality criteria, but the applied instrument helped to better assess the application. However, it was possible to consider the quality criteria in the developmental process of an medical app for the field of otorhinolaryngoglogy. In summary, the present work provide a comprehensive insight into the topic "Apps in Otorhinolaryngology" with the aim to use these modern aids in a beneficial way. 


\section{Inhaltsverzeichnis}

2.1 Mobile Health

2.2 Medizinische Apps in der HNO-Heilkunde

2.3 Wirksamkeit und Nutzen von medizinischen Apps

$2.4 \quad$ Die App als Medizinprodukt

2.5 Haftungsprävention

2.6 Die "gute“ medizinische App

2.7 Herausforderungen

2.8 Partizipation am Wandel

$2.9 \quad$ Fazit

3. Literaturrecherche zu „Apps in der HNO Heilkunde“

3.1 Auswertung der Literaturrecherche

3.2 Fazit

4. Umfrage zu „Apps in der HNO-Heilkunde“

4.1 Konzept der Umfrage

4.2 Überblick über die bewerteten Apps

4.3 Auswertung des Fragebogens

$4.4 \quad$ Fazit

5. Entwicklung einer App zum Training der mimischen

Muskulatur bei Fazialisparese
$5.1 \quad$ Konzeptionierung

5.2 Auswahl des Themas

5.3 Struktur und Aufbau der App

266

5.4 Grafische Gestaltung und Programmierung der App

266

5.5 Weitere Entwicklung und Validierung 266

$5.6 \quad$ Fazit

266

6. Zusammenfassung und Ausblick

\section{Einleitung}

Der Gebrauch von Smartphones und Tablet-PCs nimmt seit Jahren kontinuierlich zu. $81 \%$ der Deutschen ab dem 14. Lebensjahr nutzen zum aktuellen Zeitpunkt diese Technologie, die neben dem Telefonieren immer weitere Funktionen bietet [1]. Die Internetvernetzung eröffnet die Möglichkeit der Nutzung mobiler Informations- und Kommunikationstechnologie. Die Nutzung dieser für medizinische und gesundheitsnahe Dienstleistungen, “Mobile Health” (kurz: „mHealth“) genannt, gewinnt immer mehr an Bedeutung. Wie in vielen anderen Bereichen auch, hat sich im Medizinsektor eine spezielle Form der Anwendungssoftware für die Geräte bzw. ihre Betriebssysteme etabliert, die sogenannten „medizinischen Apps“.

Diese Anwendungen bieten ein breites Spektrum von vergleichsweise einfachen „Fitness“- und „Wellness“-Anwendungen bis hin zu anspruchsvollen Programmen zur Diagnostik und Therapie. Auch im Bereich der HNO-Heilkunde entstand in den letzten Jahren ein immer größer werdendes Angebot von Apps, welches sehr viele Aspekte des Faches einschließt.

Im vorliegenden Referat soll ein Überblick über den Bereich „Apps in der HNO-Heilkunde“ gegeben werden. Einschränkend muss hier klargestellt werden, dass aufgrund des sehr schnelllebigen Marktes und der mangelhaften Informationspolitik der Storebetreiber und Mobilplattformen bereits die Erfassung aller auf dem Markt befindlichen Apps unmöglich ist und insbesondere die wissenschaftliche Recherche und Auswertung deutlichen Restriktionen unterliegt. Dennoch soll durch die Auswertung möglichst vieler Themenberei- che versucht werden, ein breites Verständnis über den aktuellen Stand zu vermitteln, insbesondere auch im Hinblick auf die Chancen und Risiken solcher Anwendungen in der HNO-Heilkunde.

Im folgenden Kapitel wird in die Thematik „Mobile Health und Medizinische Apps“ eingeführt und die Wirksamkeit und der Nutzen medizinischer Apps kritisch diskutiert. Darüber hinaus wird auf die unterschiedliche Qualität der Anwendungssoftware eingegangen und erläutert, welche Kriterien eine „gute App“ erfüllen sollte. Dies schließt die Aspekte „App als Medizinprodukt“ und Haftungsfragen ebenso mit ein wie die Bedeutung der aktiven Teilnahme an solchen neuen Prozessen zum Wohle der Patienten. Darüber hinaus werden die Entwicklung der Anzahl und die Qualität von Apps in der HNOHeilkunde anhand eines exemplarisch gewählten App Stores dargestellt.

Im 3. Kapitel werden die Ergebnisse einer Literaturrecherche hinsichtlich publizierter Daten zu Apps in der HNO-Heilkunde dargestellt und aufgearbeitet.

Das 4. Kapitel beschäftigt sich mit der Auswertung einer eigens für dieses Referat durchgeführten Umfrage zu solchen HNO-Apps. Dabei wurden die Apps durch ausgewählte Nutzer mit einem speziellen Evaluationsbogen bewertet. Dieser enthält spezielle Items, um zu ermitteln, ob eine App wirklich die Voraussetzungen erfüllt, um sicher in der Patientenversorgung eingesetzt werden zu können.

Im letzten Kapitel wird aufgezeigt, wie eine neue App sinnvoll und zuverlässig konzeptualisiert und programmiert werden kann. Bei der Darstellung der ersten Entwicklungsschritte liegt der Schwerpunkt 
auf den notwendigen Details, damit eine neue App zuverlässig in der Patientenversorgung eingesetzt werden kann.

Durch die verschiedenen Schwerpunkte der einzelnen Kapitel soll in der Zusammenschau eine kritische Auseinandersetzung mit diesen Apps für das Fach der HNO-Heilkunde ermöglicht werden. Im gesamten Text wird weitestgehend vermieden, Name und Hersteller der Apps zu benennen, um eine Beeinflussung der späteren Auswahl durch den Nutzer zu vermeiden Es sollen vielmehr Hilfestellungen zu der Frage vermittelt werden, wie wir als HNO-Ärzte beurteilen können, ob medizinische Apps sinnvoll einzusetzen sind. Unter Berücksichtigung der beschriebenen Aspekte sollte es dann nicht schwierig sein, eine geeignete App für den Einsatz nach erfolgter kritischer Bewertung auszuwählen und für die Nutzung zu empfehlen.

\section{Einführung in die Thematik „Mobile Health und medizinische Apps"}

\subsection{Mobile Health}

“Mobile Health” (kurz: „mHealth“) steht für die Nutzung mobiler Informations- und Kommunikationstechnologie im Kontext medizinischer oder gesundheitsnaher Dienstleistungen. Gesundheits-Apps, die auf Smartphones, Tablet-PCs und anderen mobilen Formfaktoren betrieben werden, stellen die bekannteste und verbreitetste Variante von mHealth dar. Thematisch umfassen diese den medizinischen Bereich mit Prävention, Diagnostik und Therapie genauso wie den weiteren Gesundheitsbereich mit Fitness und Ernährung mit dem Ziel der Steigerung des körperlichen und psychischen Wohlbefindens. Es sind die Charakteristika der Technologie, die das mobile Konzept in anderen Bereichen zum Erfolg brachte und die nun ebenfalls in der Gesundheitsversorgung unterstützen sollen. Hierzu zählt z. B. die direkte Verfügbarkeit von Informationen am Ort des Geschehens, unabhängig von Öffnungszeiten oder dem Bargeldverkehr. Diese Flexibilität ermöglicht ein komfortables Nutzungserlebnis, das sich vollständig in den Alltag integrieren lässt. Dieses Konzept birgt allerdings auch Risiken in sich. Das fast immer notwendige Datenaufzeichnen, -speichern, -versenden und -verarbeiten kann eine Beeinträchtigung von Persönlichkeitsinteressen mit sich bringen. mHealth nimmt dabei Einfluss auf das Patienten-Arzt-Verhältnis, da beide Seiten die Technologie bereits angenommen haben und für ihre Zwecke nutzen. Es muss allerdings noch die geeignete Schnittstelle gefunden werden, die beide Welten im besten Sinne verbindet. Diverse Angebote bestehen bereits, welche sich durchsetzen werden, wird sich in der Zukunft zeigen.

\subsection{Medizinische Apps in der HNO-Heilkunde}

Es sind unzählige medizinische Apps auf dem Markt. Eine genaue Zahl anzugeben ist kaum möglich, da sich die Store-Betreiber der großen Mobilplattformen darüber ausschweigen und ein Zählen nur begrenzt möglich ist. Neben Apps, die z. B. eher die Bereiche Fitness oder Prävention bedienen, finden sich - wenn auch in geringerem Umfang - einige im Angebot, die medizinische Fragestellungen abdecken, und hierbei sind auch solche für den Bereich der Hals-NasenOhrenheilkunde enthalten. Bei einer exemplarischen Bestandsaufnahme mittels der retrospektiven semiautomatisierten App Store Analyse (SARASA) [2,3] und Auswertung in der Store-Kategorie „Medizin“ innerhalb des App Stores von Apple (Auslesedatum 14.04.2018) zeigte sich bei der Suche nach deutschsprachigen Apps mit HNO-relevanten Schlagworten ( $\triangleright$ Tab. 1) bspw. folgendes Bild: Es konnten lediglich 97 deutschsprachige Apps mit HNO-Bezug im deutschen App Store von Apple unter der Kategorie „Medizin“ identifiziert werden ( $\triangleright$ Tab. 2). Im Verhältnis zu insgesamt 5045 deutschsprachigen Apps ist das ein Anteil von gerade einmal 1,9\%. Vergleichsweise häufig waren Apps vertreten, die sich mit dem Hören auseinandersetzen, z. B. indem sie Hörgeräteakustikern oder gar den Betroffenen selbst erlauben, Hörgeräte App-gestützt zu konfigurieren und besonderen Bedürfnissen anzupassen oder bei Tinnitus Hilfestellung geben. Ebenso sind Apps vertreten, die als Nachschlagewerke zu verschiedenen HNO-bezogenen Fragestellungen ärztliches Fachpersonal oder die Patienten informieren, als Tagebücher für Allergiker dienen oder auf dem Weg zu einem gesunden Schlaf unterstützen wollen ( $\triangleright$ Abb. 1). Der Weg in die Kostenerstattung gestaltet sich für medizinische Apps grundsätzlich schwer. Eine Kostenübernahme durch die gesetzlichen Krankenversicherungen erfolgt bisher nur für 2 Apps im HNO-Bereich (Namentlich “Tinnitracks" und "Audimatch" der Sonormed GmbH).

Auf Basis der zur Verfügung stehenden Metadaten der ausgewählten Apps lassen sich ebenfalls einige Aussagen treffen. Die Hersteller der „HNO-Apps“ scheinen sich ihrer Verantwortung zu aktuellen Inhalten durchaus bewusst zu sein und versorgen in kürzeren Abständen mit Updates (Median: 4,76 Monate, IQR: 13,35) als dies bei anderen deutschsprachigen (Median: 11,07, IQR: 22,51) oder sprachunabhängigen Apps der Kategorie Medizin (Median 12,98 Monate, IQR 22,32) üblich ist Ebenso scheint der Wille zur umfangreichen Information der Anwender bei Apps mit HNO-Bezug größer: Die Länge der Store-Beschreibungen übersteigt hier mit 1467 Zeichen (IQR 1736) den bei deutschsprachigen Medizin-Apps üblichen Umfang (Median 921 Zeichen, IQR 1502) (๖ Tab. 2).

Auffällig ist zudem auch der größere Umfang der deutschsprachigen HNO-Apps: Die Dateigröße von Median 42,59 (IQR 65,74) gegenüber 24,65 MB (IQR 30,37) bezogen auf alle deutschsprachigen Apps mag u. a. durch die im häufig vertretenen Anwendungsfall Hören in den Apps integrierten multimedialen Funktionen (z. B. Abspielen von Tondateien) bedingt sein. Im Hinblick auf das Nutzerecho zu HNO-Apps fällt insbesondere auf, dass diese - positiv wie negativ

\ Tab. 1 Verwendete Schlagworte und zugehörige Treffer in der Store-Kategorie „Medizin“.

Schlagworte (regulärer Ausdruck, Perl-Syntax):

Initial ausgelesene Apps der Kategorie „Medizin“

davon mit deutschspra-chiger Beschreibung

manuell validiert hörtest | hörprüfung | dezibel | tinnitus | ohrgeräusch | otoskop | schlaf |[ge] * schnarch[ent]| (?<!ge)schwindel[ig] | dysphag|schluck[ge] * stör[unget]+ | hörgerät|allergie|allergisch

39427 Apps am 14.04.2018

5045 Apps

97 Apps 
- Tab. 2 Deskriptiver Vergleich der HNO-Apps mit dem deutschsprachigen und dem Gesamtangebot der Store-Kategorie “Medizin” (Apple App Store für iOS-basierte Apps, Auslesezeitpunkt 14.04.2018).

\begin{tabular}{|c|c|c|c|c|}
\hline & $\begin{array}{l}\text { Alle Apps in Medizin } \\
\qquad(N=39427)\end{array}$ & $\begin{array}{l}\text { Deutsche Beschrei-bung } \\
\qquad(\mathrm{N}=5045)\end{array}$ & $\begin{array}{c}\text { Auswahl HNO } \\
\text { deutsch }(N=375)\end{array}$ & $\begin{array}{l}\text { Auswahl HNO deutsch, } \\
\text { manuell validiert }(\mathrm{N}=97)\end{array}$ \\
\hline \multicolumn{5}{|c|}{ Alter in Monaten (insgesamt) } \\
\hline Median (IQR) & $28,22 / 34,89$ & $32,58 / 33,35$ & $37,61 / 39,65$ & $30,11 / 28,74$ \\
\hline \multicolumn{5}{|c|}{ Alter in Monaten (aktuelle Version) } \\
\hline Median (IQR) & $12,98 / 22,32$ & $11,07 / 22,51$ & $7,63 / 19,37$ & $4,76 / 13,35$ \\
\hline \multicolumn{5}{|c|}{ Dateigröße in MB } \\
\hline Median (IQR) & $22,56 / 36,08$ & $24,65 / 30,37$ & $38,27 / 57,60$ & $42,59 / 65,74$ \\
\hline \multicolumn{5}{|l|}{ Preis in $€$} \\
\hline Min: & 0,49 & 0,49 & 0,49 & 1,09 \\
\hline Median (IQR) & $\mathrm{n}=6838 ; 3,99 / 7,70$ & $\mathrm{n}=846 ; 3,49 / 3,20$ & $n=110 ; 3,49 / 2,93$ & $\mathrm{n}=21 ; 3,49 / 3,20$ \\
\hline Max: & 1099,99 & 499,99 & 59,99 & 42,99 \\
\hline \multicolumn{5}{|c|}{ Länge Beschreibung (Zeichen) } \\
\hline Median (IQR) & $757 / 1048,50$ & $921 / 1502,00$ & $1,757 / 1,673,50$ & $1467 / 1736,00$ \\
\hline \multicolumn{5}{|c|}{ Sterne (alle Versionen) } \\
\hline Median (IQR) & $\mathrm{n}=2581 ; 4,00 / 2,00$ & $\mathrm{n}=1681 ; 4,00 / 2,00$ & $\mathrm{n}=214 ; 4,00 / 1,50$ & $\mathrm{n}=51 ; 3,50 / 2,00$ \\
\hline
\end{tabular}

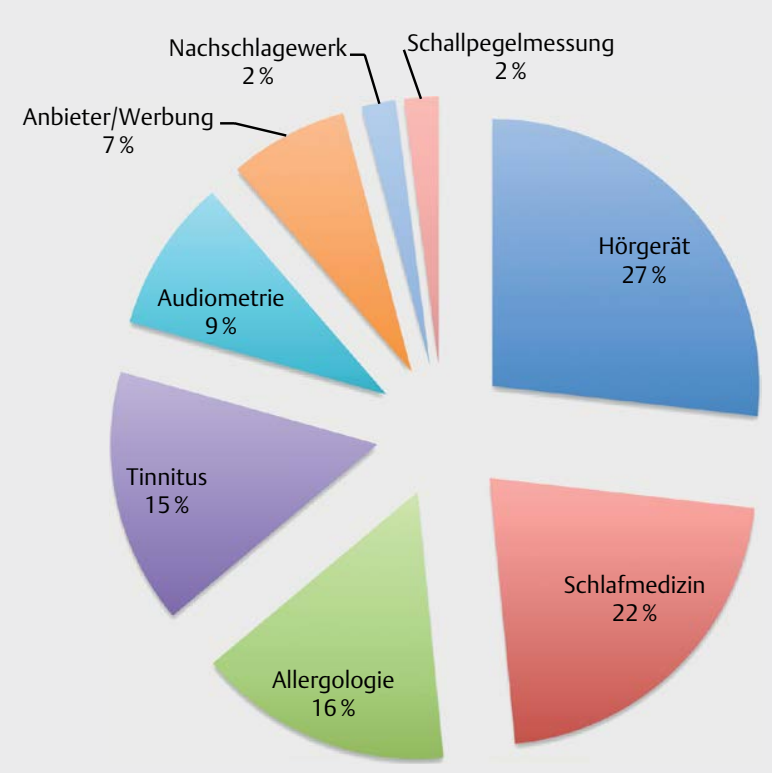

Abb. 1 Prozentuale Verteilung der im Rahmen der Schlagwortsuche identifizierten Apps zu Bereichen der HNO-Heilkunde (Apple App Store für iOS-basierte Apps, Auslesezeitpunkt 14.04.2018).

- Anwender häufiger dazu zu animieren scheinen, eine Meinung zu äußern. Zwar unterscheiden sich die Bewertungen im Median nur marginal (im Median jeweils 4 Sterne, Unterschiede bestehen hinsichtlich des Interquartil-Abstandes), jedoch ist der Anteil an Apps, zu denen überhaupt Bewertungen vorliegen mit 52,6\% (51/97) wesentlich höher als bei anderen deutschsprachigen Apps der Kategorie Medizin mit 33,3\% (1681/5045).
Etwas über jede fünfte HNO-App ist kostenpflichtig (21,6\%, 21/97), während dies nur für knapp $17 \%$ (16,7\%, 846/5045) aller deutschsprachigen Medizin-Apps der Fall ist. Dies verwundert, da aufgrund des hohen Anteils von Apps, die der Anpassung von Hörgeräten dienen und ohne diese gar nicht nutzbar sind, hier eher ein geringerer Anteil kostenpflichtiger Apps zu vermuten gewesen wäre.

\subsection{Wirksamkeit und Nutzen von medizinischen Apps}

Viele Ansätze klingen vielversprechend, doch fehlt heute noch ausreichend wissenschaftliche Evidenz, die eine positive Auswirkung von medizinischen Apps auf den individuellen Gesundheitszustand untermauern würde. Eine solche ist als Grundlage zur Refinanzierung zu fordern, insbesondere im Erstattungskontext von Krankenkassen, die an rechtliche Bedingungen zum erwiesenen Nutzen gebunden sind [4].

In der wissenschaftlichen Literatur finden sich zum Thema „Mobile Health“ zwar zahlreiche Veröffentlichungen, jedoch sind wenige von diesen auf deutsche Verhältnisse übertragbar oder auf so eng gesteckte Anwendungsbereiche fokussiert, so dass eine Übertragung auf den gesamten Sektor und generelle Schlussfolgerungen daraus unwissenschaftlich wären. Solchen Konklusionen im Überblick steht eine große Methodenvariabilität entgegen. Herkömmliche Studiendesigns setzen Untersuchungszeiträume voraus, die, mit Blick auf die kurzen Entwicklungszyklen im Smartphone-Bereich, nur bedingt anwendbar sind [4].

Abschließende Studien stehen also noch aus. Festzuhalten ist jedoch, dass Apps zur Aufnahme oder Steigerung von Fitnessaktivitäten überwiegend positiv bewertet werden. Ein Grund wird in der Unabhängigkeit von Gruppendruck gesehen, die bestimmten Zielgruppen den Einstieg erleichtert. In Frage gestellt wird die Therapietreue [5]. Jedoch bieten viele Apps Erinnerungsfunktionen und gerade sol- 
che, die spielerisch zur Nutzung animieren, individuelles Feedback zum Fortschritt geben oder auch in begrenztem Rahmen den Wettbewerb mit Freunden oder Gleichgesinnten ermöglichen, werden als besonders positiv bewertet. Dabei sollten sie den Anwender bei Misserfolgen nicht in ein schlechtes Licht rücken [6]. So gelingt die Verbesserung der Partizipation an Gesundheitsprozessen, für die Apps als Chance betrachtet werden und Patienten können unter Verwendung ihrer Smartphones einen Eigenbeitrag zur Verbesserung ihrer Gesundheit leisten [7].

\subsection{Die App als Medizinprodukt}

Genauso wie beim Zungenspatel oder dem Otoskop kann es sich auch bei medizinischen Apps um Medizinprodukte handeln. Dieser Umstand ist insbesondere für professionelle Anwender von Bedeutung, wenn es zum Schadensfall kommt, der ursächlich auf die eine App zurückzuführen ist. Keinesfalls darf der Anwender davon ausgehen, dass die App, die er vor sich hat, ein Medizinprodukt ist - auch wenn sie für einen medizinischen Zweck eingesetzt werden könnte. Oftmals sieht man der App nicht an, wofür sie gedacht ist und dem Nutzer wird die Entscheidung überlassen, wo und wie er die App einsetzt. Das ist bei Medizinprodukten anders. Der Hersteller legte im Verlauf der Produktentwicklung selbst fest, ob es sich bei einem Produkt um ein Medizinprodukt handelt, indem er bspw. einer App eine medizinische Zweckbestimmung entsprechend dem Medizinproduktegesetz zuweist [8]. Das bedeutet im Wesentlichen, dass der Hersteller einen Zweck für seine App im Bereich d Diagnostik, Therapie, Prävention oder Verhütung vorgesehen hat (entsprechend $\S$ 3 Medizinproduktegesetz MPG ).

Diese Entscheidung hat weitreichende und kostenaufwändige Konsequenzen, denn der Hersteller muss nun ein mehr oder weniger aufwändiges Verfahren bis zu Konformitätserklärung durchlaufen. Mit letzterer bestätigt er, dass er die "grundlegenden Anforderungen” der einschlägigen EU-Verordnung (Medical Device Directive - MDD [9]) erfüllt. Erst nach der Konformitätserklärung darf eine CE-Kennzeichnung angebracht und das Produkt in den europäischen Markt eingeführt und somit an Dritte abgegeben werden. Abhängig von der Risikoklasse des Medizinprodukts (I, Im, Is, Ila, IIb und III) muss eine sogenannte „benannte Stelle“ hinzugezogen werden, die bspw. die Gültigkeit von Messfunktionen der App (ab Risikoklasse Im) bescheinigt. Apps, die ein Medizinprodukt darstellen, müssen daher die grundlegenden Anforderungen nach Dokumentation, Softwareentwicklung, Tests, Validierung, Gebrauchstauglichkeit, Risikomanagement und Versionsmanagement dem Gesetz entsprechend erfüllen. Hersteller von Apps, die keine Medizinprodukte sind, haben keine Verpflichtung dazu. Zur ausführlichen Darstellung sei auf die CHARISMHA-Studie und weiterführende Literatur verwiesen [10,11]. Es ist nachvollziehbar, dass sich eine Vielzahl von Herstellern aufgrund des Aufwands bewusst gegen die Deklaration ihrer App als Medizinprodukt entscheidet. Einer noch größeren Anzahl an Herstellern wird die Problematik gar nicht bewusst sein, da sie aus anderen Entwicklungsbereichen stammen. Die Konsequenzen für die Anwender, insbesondere in professionellen Bereichen, sind allerdings weitreichend.

\subsection{Haftungsprävention}

Der Arzt schuldet seinen Patienten laut dem Bürgerlichen Gesetzbuch (BGB) § 630 a eine nach allgemein akzeptierten fachlichen Standards anerkannte Behandlung, soweit es nicht anders vereinbart wurde. Entsprechend können Schadensersatzansprüche gegenüber dem Arzt geltend gemacht werden, wenn dieser ungeeignete Instrumente oder Methoden, welche schadensursächlich waren, am Patienten eingesetzt hat. Apps bergen aufgrund der wenig einheitlichen Entwicklungspraxis und kaum vorhandenen staatlichen Überwachung ein erhöhtes Haftungsrisiko für ihre Anwender und nicht für die Hersteller [12]. Nicht zwangsläufig kann der Hersteller haftbar gemacht werden. Insbesondere wird es dann schwierig, wenn letzterer der App keine medizinische Zweckbestimmung (s. o.) gegeben hat, diese aber im medizinischen Kontext eingesetzt wurde. Doch auch dann, wenn die App ein Medizinprodukt nach dem Medizinproduktegesetz ist, hängt es noch vom bestimmungsgemäßen Einsatz durch den Anwender ab. Problematisch wird es nämlich, wenn die App abweichend von ihrer Zweckbestimmung einsetzt wurde und es hieraus ursächlich zum Schaden für den Patienten kommt. In der Regel wird der Hersteller hier die Verantwortung ablehnen. Im Sinne der Haftungsprävention soll sich der Arzt vor dem Betrieb und der Anwendung am Patienten gut über die App informieren. Das schließt ihre Zweckbestimmung, Funktionalität, ihren Anwendungsbereich und Anwendungsbeschränkungen, ihr Risikopotenzial usw. ein [12]. Dringend zu empfehlen ist die inhaltliche Auseinandersetzung mit App-Beschreibungen und Gebrauchsanweisungen (insbesondere bei Medizinprodukten). Ferner sollen die Apps vorher getestet werden, indem Proberechnungen, Probemessungen und Vergleiche mit Standardmethoden durchgeführt werden, um die Plausibilität zu prüfen und einen praktischen Eindruck von der Validität der App zu bekommen. [12]. Darüber hinaus sollten mögliche, zumindest aber wahrscheinliche Anwenderfehler bedacht und deren Konsequenzen auf die Anwendung der App überprüft werden. Die App muss letztendlich den Anwender von ihrer Eignung überzeugen - bei Ungereimtheiten muss hier die Entscheidung gegen die Anwendung erfolgen, um haftungspräventiv tätig zu werden.

\subsection{Die „gute“ medizinische App}

Der Anteil an Apps, die vom Hersteller eine medizinische Zweckbestimmung zugewiesen bekommen haben, also ein Medizinprodukt sind, und mit dieser auch im App Store zu finden sind, ist sehr gering. Für Apps im deutschen App Store von Apple, mit deutschen Beschreibungstexten in den Kategorien „Medizin“ und „Gesundheit und Fitness“, konnte in einer kürzlich durchgeführten Untersuchung gerade einmal bei 34 von 8767 Apps ein solcher Hinweis in den Beschreibungstexten identifiziert werden [2]. Für andere „Qualitätssiegel“ war das Ergebnis mit 5 von 8767 Apps noch bescheidener, obwohl 13 Initiativen gezählt wurden, die Qualitätssiegel anbieten und das Angebot daher als gut anzusehen ist. Die „CE-Kennzeichnung“ ist in dem Sinne kein „Qualitätssiegel“, sondern stellt bei Medizinprodukten eine gesetzlich vorgeschriebene Notwendigkeit dar. Die geringe Anzahl in dieser Weise zertifizierter Apps weist darauf hin, dass für die Nutzer bei der Mehrzahl der Apps keine Sicherheit bezüglich der Qualität gegeben ist. Lediglich eine einzige medizinische App im HNO Bereich mit therapeutischem Ansatz (Tinnitracks) erfüllt diese gesetzlich vorgeschriebene Anforderung („CE-Kennzeichnung“) und wird gleichzeitig von einer Vielzahl von Krankenkassen angeboten. Diese App kann erst nach erfolgter Diagnostik und nur durch den teilnehmenden HNO-Arzt verordnet werden. In dieser Konstellation scheint das Zusammenspiel aller beteiligten Akteure innerhalb des 
Gesundheitssystems zum Einsatz der App zu funktionieren. Weitere Apps desselben Herstellers werden oder sollen offenbar in einem ähnlichen Setting in Kooperation mit den Krankenkassen folgen, etwa eine App zum Screening der Hörfähigkeit bzw. eine zum Einsatz im Rahmen des Tinnitus-Counselings [13].

Für Anwender ist es sehr schwierig, eine qualitativ hochwertige App zu identifizieren. Grundsätzlich stellt sich die Frage, was eine "gute“, „qualitativ hochwertige“ App ausmacht. Der Eindruck einer „guten“ App entsteht für deren Anwender u. a. dadurch aus, dass das Nutzungserlebnis ihnen zu jedem Zeitpunkt das Gefühl vermittelt, mit einer sorgfältig entwickelten Anwendung zu arbeiten. Subjektiv zeigt sich das ab dem ersten Moment der Nutzung häufig dadurch, dass zuerst individuelle Parameter abgefragt werden und diese in der weiteren Nutzung als Berechnungsgrundlage dienen, sodass durch die App errechnete Größen oder Empfehlungen sowohl nachvollziehbar als auch für den einzelnen Nutzer angemessen erscheinen. Die Anwendung sollte intuitiv erfolgen können und jede Funktion plausibel, bzw. durch gut zugängliche, übersichtliche Erläuterungen zu erschließen sein. Besonders ein flexibles Reagieren der App auf Veränderungen, z. B. wenn der Nutzer das Idealsetting für die Anwendung der App verlässt und die App dann von sich aus darauf hinweist und Empfehlungen zur Reaktion auf die veränderte Situation gibt, weist auf eine gut geplante Entwicklung, realistische Testung und sorgfältige Rückführung von Testerkenntnissen in die Weiterentwicklung hin. Auf diese Weise schließen gut entwickelte Apps, soweit möglich, eine Gefährdung für die Gesundheit des Nutzers aus. Eine „gute App“ kann so ihren Zweck vollständig, effizient und zuverlässig erfüllen und den Nutzer zufriedenstellen, idealerweise sogar indem sie seine Erwartungen übertrifft.

Wo Risiken präventiv ausgeschlossen werden können, können sie auch missachtet werden. „Schlechte“ Apps weisen Folgen ungenügender Sorgfalt in ihrer Entwicklung auf, die im schlimmsten Fall den Nutzer gefährden können, in jedem Fall aber zu Frust bei der Anwendung führen. Ist eine App, die als Teil einer Therapie eingesetzt werden soll „schlecht“, oder tendiert zumindest eher in diese Richtung, statt Aspekte einer „guten“ App aufzuweisen, ist mit einer geringen Adhärenz zu rechnen. Aufgrund des unübersichtlich großen Angebots an Apps und der Intransparenz über deren Qualität wird bei Kunden, in unserem Fall Patienten, aber auch aus der Politik von Fachverbänden und Verbraucherschützern der Ruf nach Kontrollinstanzen lauter, die fachkundig und ohne Interessenkonflikte eine Prüfung und Bewertung vornehmen. Eine Prüfung durch staatliche Instanzen findet nur in eng gestecktem Rahmen statt und betrifft lediglich einen sehr geringen Anteil aller Apps. Prüfstätten und Zertifizierungseinheiten erkennen das große Potenzial in der Unsicherheit über die Qualität von Apps und bieten Zertifizierungen an, legen aber ihre Prüfverfahren nicht offen, sodass nicht pauschal festgestellt werden kann, dass allgemein gültige Testgütekriterien erfüllt wurden und „besiegelte“ Apps alle Qualitätskriterien erfüllen.

\subsection{Herausforderungen}

Apps, die im medizinischen Kontext angeboten werden, weisen teilweise inakzeptable Qualitätsdefizite auf. Kennzeichnend ist zum Einen die Überschätzung der technischen Möglichkeiten von Smartphones, wie sie beispielhaft am Smartphone-gestützten MelanomScreening zu erkennen war, das sehr früh eine an sich gute Idee auf einen Technologieträger übertragen hat, der schlichtweg noch ungeeignet war [14]. Zum anderen wittern auch Hersteller aus gesundheitsfernen Bereichen Chancen, diesem Markt über Apps im Gesundheitskontext beizutreten, vernachlässigen aber aufgrund mangelnder Erfahrung und Expertise in der Herangehensweise branchenübliche Standards und sogar regulatorische Vorgaben. Aus einem weniger von Zweifeln und Risikoängsten geprägten Blickwinkel betrachtet tritt jedoch die Besonderheit der Situation ans Licht: Neben der fast flächendeckenden Verfügbarkeit der, zumindest in genau definierten Rahmen geeigneten Technologie, wohnt dem Markt der Mobile Health Anwendungen ein Gründergeist inne, der unserem Gesundheitssystem viele Chancen und eine Basis für neue Lösungsansätze bietet. Um nachhaltigen Erfolg zu erleben sind Hersteller durchaus daran interessiert, hochwertige und durchdachte Produkte anzubieten. Für die notwendige Sorgfalt im medizinischen Bereich und das unabdingbare Beachten und Weiterführen von „Best Practice“ -Methoden müssen Hersteller angeregt und sensibilisiert, im besten Fall dabei unterstützt werden.

\subsection{Partizipation am Wandel}

Wie die letzten beiden Ärztetage gezeigt haben, ist das Bewusstsein zur Notwendigkeit der Digitalisierung in der Medizin - und der eigenen Beteiligung daran - inzwischen auch in der Ärzteschaft gestiegen. Zunehmend werden bei berufsständischen Organisationen, Fachgesellschaften und Berufsverbänden Arbeitsgruppen bzw. Task Forces initiiert, die sich mit digitalen Fragestellungen und einer möglichen Mitgestaltung auf deren Implementierung in der Gesundheitsversorgung auseinandersetzen. Auf welche Weise diese Auseinandersetzung erfolgt und wie weit die jeweiligen Bemühungen bereits gediehen sind variiert jedoch je nach Beteiligten stark. Manche Gesellschaften setzen sich im App-Kontext bspw. mit der Vergabe von Gütesiegeln auseinander. Die Deutsche Gesellschaft für Innere Medizin unterstützt hingegen aktiv die Entwicklung eines Werkzeugs (SARASA), das der formalen Filterung von Apps, die in den Stores der großen Mobilplattformen gelistet sind, dienen soll [3].

Als hilfreich können auch Bemühungen angesehen werden, fachgesellschafts-übergreifend Qualitätskriterien abzustimmen. Hierzu gehören auch gemeinschaftlich festzulegende Kriterien zur Identifikation geeigneter Apps und deren Evaluation, die zudem geeignet an alle Beteiligten (Anwender ebenso wie Anbieter und Entwickler) kommuniziert werden müssen [15]. Dies dient auch der Transparenz: Für Hersteller sind so die Voraussetzungen und Bewertungsmaßstäbe, unter denen die Ärzteschaft überhaupt bereit wäre, eine Nutzung von Apps in Betracht zu ziehen, schnell ersichtlich. Dies kann die Basis für eine qualitätsgesicherte Entwicklung im Lebenszyklus der App sein [15]. Eine Kongruenz zwischen den verschiedenen Gesellschaften kann sicherlich zu grundlegenden Aspekten wie ethischer Unbedenklichkeit, inhaltlicher Validität, Risikoangemessenheit, Transparenz und Zweckmäßigkeit ebenso wie eher technischen Faktoren, z. B. technischer Angemessenheit, Gebrauchstauglichkeit, Ressourceneffizienz und Sicherheitsbelangen erreicht werden. Es soll darauf hingearbeitet werden, dass einzelne Gesellschaften sich nicht selbst mit der - schwerlich für sie zu leistenden - technischen Prüfung dieser Produkte auseinandersetzen müssen, sondern vielmehr die nötigen Prozesse zur durchzuführenden validen Evaluation, die dann von geeigneten Partnern durchgeführt werden kann, abge- 
stimmt werden. Individuelle (fachspezifische) Anforderungen bzw. Schwerpunkte der einzelnen Fachgesellschaften und Berufsverbände bleiben von der übergreifenden Abstimmung jedoch unberührt [15]; vielmehr geht es darum, dass alle Stakeholder einen Beitrag zur Qualitätssicherung leisten können und ihnen hierfür die passenden Werkzeuge bereitstehen.

\subsection{Fazit}

Bei dafür affinen Patienten - und deren Zahl wächst - ist Mobile Health eine patientennahe Technologie: Sie eröffnet vergleichsweise einfach Möglichkeiten, Diagnostik in lebenstypischen Situationen durchzuführen, die Therapie engmaschiger zu begleiten und dabei perspektivisch unnötige Wege zu vermeiden. Gleiches kann für die HNO angestrebt werden. Für eine hohe Adhärenz sind nicht nur eine hohe Genauigkeit und eine aus medizinischer Sicht sorgfältige und vollständige Umsetzung der Funktion notwendig. Eine hohe Qualität bedeutet in diesem Kontext auch, dass die Barriere zum Patienten durch ein intuitives und flexibel reagierendes Bedienkonzept niedrig gehalten wird. Ein eindeutiger wissenschaftlicher Beleg für einen positiven Nutzen des Einsatzes von Smartphone-Apps im medizinischen Kontext steht noch aus. Das kann aber durchaus auf die schwierige Anwendbarkeit etablierter Methoden in diesem sich schnell entwickelnden Bereich zurückgeführt werden.

Die Umsetzung von Apps aus dem Gesundheitsbereich lässt vielfach die Beachtung von Qualitätskriterien vermissen, weshalb an dieser Stelle zur Mitgestaltung im Sinne der Ärzteschaft und im Sinne des Patientenwohls aufrufen werden soll. Das sehr große Angebot von Apps lässt eine Instanz zu deren übersichtlicher Bewertung vermissen. Eine Deklaration einer App als Medizinprodukt durch den Hersteller ist eine mögliche Option, die (zumindest mehr) Sicherheit gibt, dieser Weg wird aber von den wenigsten Herstellern gegangen. Möchten wir als Ärzte auf Mobile Health setzen, kommen wir schon allein aus Haftungsgründen nicht darum herum, uns mit verwendeten Apps ausgiebig und sorgfältig auseinanderzusetzen. Mit ärztlichen Anwendern, die sich unter sorgfältiger Einarbeitung auf die Technologie einlassen und so selber an der Qualität von Mobile Health Anteil haben, mit fächerübergreifend durch Fachgesellschaften und Berufsverbände abgestimmten Qualitätskriterien und mit der Beachtung dieser durch die Hersteller bedeutet Mobile Health einen Gewinn für die genannten Beteiligten, eine Entlastung für das solidarische Gesundheitssystem und idealerweise eine Stärkung des Gesundheitsbewusstseins des Patienten.

\section{Literaturrecherche zu „Apps in der HNO Heilkunde“}

Zur Evaluierung der wissenschaftlichen Auseinandersetzung mit dem Thema „Apps in der HNO Heilkunde“ erfolgte Ende März 2018 eine Literaturrecherche auf der textbasierten englischsprachigen Metadatenbank Pubmed (https://www.ncbi.nlm.nih.gov/pubmed). Hierfür wurden die Begriffe „app“, „mobile app“ und „mobile device“ mit verschiedenen Fachtermini aus dem HNO-Bereich kombiniert.

\subsection{Auswertung der Literaturrecherche}

Es konnten 71 Publikationen identifiziert werden, wobei im Jahre 2013 eine Studie, 20145 Studien, 201511 Studien, 201619 Studi- en und 201727 Studien publiziert wurden. In den Monaten Januar bis März 2018 sind bereits 8 veröffentlicht worden. Die gefunden Studien konnten 14 Bereichen aus dem HNO-Spektrum zugeordnet werden. In $>$ Abb. 2 sind ein Überblick über die 14 definierten Bereiche und deren prozentuale Verteilung (A) sowie die Anzahl an Studien pro Publikationsjahr (B) dargestellt. Im Folgenden werden die einzelnen Bereiche mit den zugehörigen Studien erläutert.

\subsubsection{Studien zu Apps als Audiometer}

Zur Thematik „Apps als Audiometer“ konnten 11 Studien gefunden werden [16-26]. Meist wurden Apps untersucht, in denen die audiometrische Funktion des Smartphones mit normal durchgeführten tonaudiometrischen Tests verglichen wurden [16-22, 24-26]. Nur eine Publikation beschrieb eine App für einen sprachaudiometrischen Test [23]. Die Genauigkeit der auf dem Smartphone durchgeführten audiologischen Unter-suchungen wurde in einigen Arbeiten mit Abweichungen von $10 \mathrm{~dB}$ [26] bis zu $25 \mathrm{~dB}$ beschrieben [17]. Das Smartphone wurde jedoch teilweise im Freifeld eingesetzt, was die Genauigkeit einschränkt [17]. Andere Autoren beschrieben eine hohe Genauigkeit der Smartphone Apps [16, 20], insbesondere beim Einsatz von Kopfhörern [22]. Ein professionelles Setting beim Einsatz der Anwendungssoftware erzielte ein höheres Maß an Genauigkeit im Vergleich zu einem vom Nutzer gewählten Setting [16, 20]. In der Arbeit, in der das Sprachverständnis untersucht wurde, ergab sich eine hohe Übereinstimmung der mit dem Smartphone und mit den

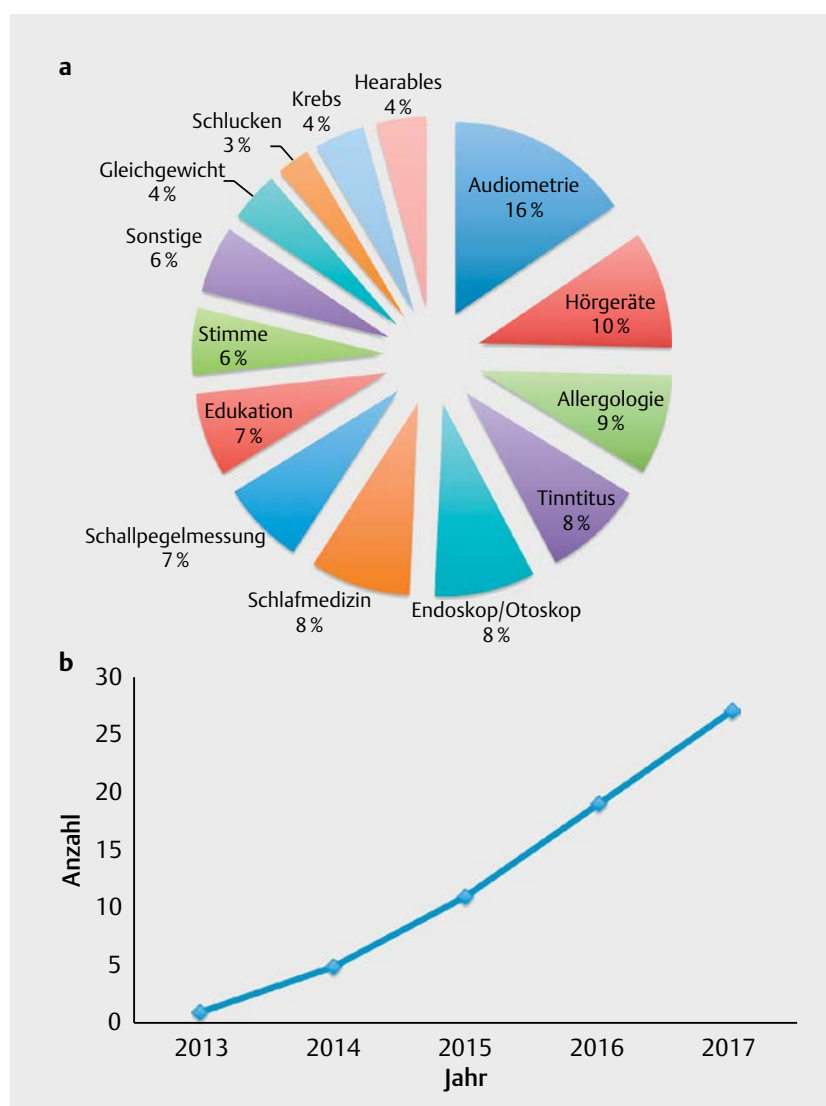

- Abb. 2 A: Zuordnung der ermittelten Studien über medizinische Apps zu Bereichen der HNO-Heilkunde und deren prozentuale Verteilung. B: Zuordnung der Studien zum Publikationsjahr. 
durch das Audiometer gemessenen Sprachverständlichkeitswerten $(r=0,89)$ [23]. In 2 Studien wurden verschiedene audiometrische Apps bzgl. der Evaluation einer Hörstörung bei älteren Patienten $[17,18]$ bzw. der möglichen Detektion eines akuten Hörverlustes untersucht [20]. Trotz teils hoher Korrelation der gemessenen Werte mit denen von zertifizierten Geräten wurde darauf hingewiesen, dass sich eine klinische Entscheidung in Bezug auf eine Therapie weiterhin auf zuverlässige und geprüfte klinische Parameter stützen sollte. Ein Review zu der Thematik „Apps als Audiometer“ aus dem Jahre 2016 identifizierte 30 Apps mit einer audiometrischen Funktion, von denen nur 6 im Rahmen von Studien vorab überprüft worden waren. Die Autoren kamen daher zu der Einschätzung, dass diese Art von Apps gute Hilfsmittel sein können, aber auf Grund der nicht existenten oder differierender Studienergebnisse und beschriebener teils fehlerhafter Funktion weiterhin nur klinisch erprobte und zertifizierte Geräte zur Diagnostik eingesetzt werden sollten [27].

\subsubsection{Studien zu Apps mit Schallpegelmess-Funktion}

Fünf Autoren untersuchten die Messung von Schalldruckpegeln mit Hilfe von Apps in Smartphones [28-32]. In einer Arbeit wurde auch der Einsatz eines Apple iPod als Gerät beschrieben [32]. Die Genauigkeit der Messungen wurde in den Studien von „zufriedenstellend“ $[13,14]$ bis „mit erheblichen Abweichungen“ $[15,16]$ bewertet. In einer Publikation konnte gezeigt werden, dass der zusätzliche Einsatz eines Mikrophons zu einer höheren Genauigkeit der Messungen führt [30]. Der iPod war wie die anderen Geräte geeignet die durchschnittliche Lärmbelastung während des Arbeitstages zu messen. Allerdings waren konventionelle Pegelmesser genauer [32].

\subsubsection{Studien zu Apps des Themenbereiches Edukation}

Zu dieser Thematik konnten 5 Studien mit unterschiedlichen Aspekten identifiziert werden [33-37]. Zwei Arbeiten befassten sich mit Apps, die eine Verbesserung der Ausbildung von HNO-Assistenten zum Ziel hat. Eine App war entwickelt worden um mit anderen Nutzern spezifische Inhalte zu teilen und anderen Nutzern Fragen zu stellen [34]. Diese App wurde vermehrt von jungen, in der Ausbildung stehenden Mitarbeitern genutzt. Die Teilnehmer zeigten zwar nach einem Jahr der Nutzung einen besseren Ausbildungsstand als zu Beginn, jedoch fehlte in der Arbeit der Vergleich mit einer Kontrollgruppe. Wiederum ohne Kontrollgruppe wurde in einer anderen Studie der Einsatz einer Quiz-App mit Bildern unterschiedlicher PathologieBefunde des Trommelfells untersucht [37]. Nach Nutzung der App wurde ein besseres Verständnis der Pathologien des Trommelfells beschrieben als vor dem Einsatz der Quiz-App. In einer weiteren Arbeit wurde bei US-amerikanischen HNO-Ärzten nach dem Bedarf einer für das Smartphone optimierten Homepage und/oder App in Bezug auf Informations- und Literaturrecherchen gefragt. Auf der Basis dieser Daten wurde eine Homepage erstellt, die in Zukunft auch als App erhältlich sein soll [33]. In einer Publikation wurde der Einsatz von Smartphones zur Gesundheitsschulung in Bezug auf das Tragen von Gehörschutz bei Farmarbeitern evaluiert [35]. Es konnte keine Überlegenheit der App-basierten Schulung im Vergleich zur klassischen persönlichen Schulung aufgezeigt werden. Eine Zusammenfassung über den Einsatz von Apps im Bereich „Hören“ bis zum Jahr 2015 ist einer in diesem Jahre veröffentlichte Übersichtsarbeit zu entnehmen [36], in der sowohl Apps für Personen im Gesundheitswesen als auch solche für Patienten beschrieben wurden.

\subsubsection{Studien zu Apps mit dem Thema Tinnitus}

Zu diesem Themenbereich wurden insgesamt 6 Publikationen gefunden [38-43]. Einerseits wurden Apps untersucht, die im Rahmen einer Tinnitus-Retraining-Therapie eingesetzt werden können. Die Nutzung dieser Apps hatte einen positiven Effekt, denn die Belastung durch den Tinnitus konnte reduziert werden [39, 43]. Andererseits wurden Apps untersucht, mit denen sowohl das Vorkommen als auch die Belastung durch einen Tinnitus untersucht werden kann. Dabei zeigten die Apps eine gute Anwendbarkeit bei der longitudinalen Beschreibung von Symptomen [41], aber auch der Umgang mit dem Tinnitus konnte durch eine Nutzung dieser App verbessert werden [38]. In anderen Arbeiten wurde untersucht, ob das Tinnitus pitch masking mit Apps für den Patienten vereinfacht und verbessert werden kann. Dies konnte in den Studien tatsächlich nachgewiesen werden $[40,43]$. In einer weiteren Publikation wurde untersucht, ob über Apps eine Rekrutierung von Tinnitus-Patienten zur Teilnahme an Studien erfolgen kann Dies war zwar prinzipiell möglich, aber mit klassischen Rekrutierungsmethoden konnte eine homogenere Gruppe von Patienten erzielt werden [42].

\subsubsection{Studien zu Apps mit der Funktion „endoskopische“ und "otoskopische" Bildgebung}

Aufgrund der inzwischen sehr guten Kameras von Smartphones können diese als Ersatz oder Zusatz zum klassischen Endoskop eingesetzt werden. Dafür muss jedoch immer ein Spezialadapter zur Befestigung an das klassische Endoskop oder Otoskop eingesetzt werden. Es konnten insgesamt 6 Studien identifiziert werden, in denen der Einsatz eines Smartphones für eine Bildgebung im HNO-Bereich genutzt wurde [44-48]. In 2 Arbeiten wurde die Qualität der gewonnenen Bilder mit klassischen Verfahren verglichen. Es konnte in beiden Studien eine hohe Qualität der App-basierten Bildgebung gezeigt werden, was für den Einsatz des Smartphones mit Endoskop in der klinischen Routine spricht [47, 48]. In einer weiteren Publikation wurde der mögliche Einsatz der Geräte durch Laien, z. B. von Eltern, für telemedizinische Befunde beschrieben [45]. Es ergab sich jedoch eine deutlich geringere Aussagekraft der Bilder bei der Bilderstellung durch Laien im Vergleich zu professionellen Anwendern. [46]. Eine weitere Studie enthält Daten zur Entwicklung einer Smartphone-assistierten Bildgebung. Diese enthält auch einen Überblick über die auf dem Markt vorhandenen Systeme [44].

\subsubsection{Studien zu Apps zum Thema Schlafmedizin}

Zu diesem Themenbereich konnten insgesamt 6 Studien identifiziert werden [49-54] In einer Pilotstudie konnte die effektive Messung von Schnarchen und weiteren Symptomen einer obstruktiven Schlafapnoe mit Hilfe einer App während einer schlafmedizinischen Untersuchung gezeigt werden. Die häusliche Anwendung hingegen wurde als problematisch beschrieben, da diese mit erheblichen Störfaktoren behaftet sein kann [51]. In einer weiteren Arbeit wurde untersucht, ob die Schlafdauer durch den Einsatz einer Schulungs- und Monitoring-App verbessert werden kann. Dies war bei einigen Probanden möglich [55]. Die Besserung eines obstruktiven Schlafapnoe 
Syndrom (OSAS) durch eine Gewichtsreduktion unter App-Kontrolle wurde auch untersucht. Die Nutzer der App erzielten eine Gewichtsreduktion, diese hatte jedoch keinen Einfluss auf das OSAS [50]. In den 4 Reviews zu Apps zum Thema „Schlafen und Schnarchen “ [49, 52-54] wurde der undurchsichtige Umgang mit den erhobenen Daten kritisiert, außerdem dass für die Analysen und Aussagen der Apps häufig keine wissenschaftliche belegte Evidenz angegeben bzw. zu recherchieren war.

\subsubsection{Studien zu Apps zum Thema Stimme}

In 4 Publikationen wurden Apps im Bereich der Stimme aufgearbeitet [56-59]. In 2 Arbeiten wurde gezeigt, dass über eine solche App ein multidimensionales Stimmprofil erstellt werden kann. Dabei wurde untersucht, wie präzise verschiedene Parameter der Stimmqualität mit Hilfe der App ermittelt werden können. Die Autoren kamen zu dem Ergebnis, dass die Apps vergleichbare Ergebnisse wie konventionelle Geräte liefern können und gut in einem klinischen Setup eingesetzt werden können $[56,58]$. In einer weiteren Studie wurde gezeigt, dass die Qualität der Mikrophonaufnahmen mit Smartphones vergleichbar ist mit denen von Mikrophonen, die normalerweise im Rahmen von Studien zur Stimmqualität einsetzt werden [59]. In einer weiteren Arbeit wurde untersucht, ob die Stimmqualität von Studenten, die einen Vortrag halten mussten, mit einer solchen App gemessen und kontrolliert werden kann. Die Studie war zum Zeitpunkt der Publikation zwar noch nicht abgeschlossen, aber erste Ergebnisse zeigten, dass die App erfolgreich eingesetzt werden kann um die Qualität der Stimme zu erfassen [57].

\subsubsection{Studien zu Apps zum Thema Gleichgewicht}

Zu diesem Thema wurde 3 Studien gefunden [60-62]. In einer Studie wurde eine App bewertet, mit der Unterberger Tretversuch erfasst und ausgewertet werden kann. [62]. Demnach kann über das vom Probanden gehaltene Smartphone die Winkelabweichung so exakt messen wie es für die klinische Routine empfohlen wird. Ebenso wird eine weitere App positiv bewertet, mit der die visuelle Vertikale bei pädiatrischen Patienten gemessen werden kann [60]. Eine andere Publikation beschäftige sich mit dem Befreiungsmanöver bei Lagerungsschwindel. Die Autoren kamen zu dem Schluss, dass die betreffende App beim Erlernen der eigenständigen Durchführung dieser Befreiungsmanöver hilfreich sein kann. [61].

\subsubsection{Studien zu Apps zum Thema Schlucken}

In 2 Studien wird die Entwicklung [63] und die Überprüfung [64] von Apps zur Minderung einer Dysphagie nach Tumortherapie beschrieben. In der ersten Arbeit wurden auf der Basis von strukturierten Interviews mit Betroffenen wesentliche Funktionen einer App zur Therapie einer Dysphagie identifiziert [63]. Diese beinhalten ein Biofeedback, eine klare Beschreibung der Pathologie und die Anleitung zu Übungen. In der zweiten Publikation wurde gezeigt, dass der Einsatz einer App zur Unterstützung der Therapie einer Dysphagie von den Betroffenen als sehr positiv eingeschätzt wird, was durch ein positives Datalogging bestätigt wurde [64].

\subsubsection{Studien zu Apps zum Themenbereich Hörgeräte}

Zu dieser Thematik fanden sich insgesamt 7 Arbeiten [65-71]. In 5 Studien wurde untersucht, ob die Mikrophontechnologie des Smartphones genützt werden kann, um das Sprachverstehen zu verbes- sern [69, 70]. In 2 Publikationen wurde der Einsatz von speziellen Apps mit Rauschunterdrückungsalgorithmen zur Verbesserung des Sprachverstehens untersucht $[68,71]$. In allen diesen Studien konnte eine experimentelle Verbesserung des Sprachverständnisses gezeigt werden, in einigen wurde dieser Effekt auch an Probanden bestätigt. Ob diese Technologien schon den Einsatz in aktuelle Hörgeräte gefunden haben, wurde in den Studien nicht beschrieben. Im Rahmen einer weiteren Studie wurde evaluiert, inwieweit der Einsatz neuer Technologien im Bereich der Hörgeräte und Smartphones die Arzt-Patienten-Beziehung beeinflussen kann [67]. Hierzu wurden Fälle konstruiert und strukturierte Interviews mit Ärzten und Patienten geführt Der Einsatz der neuen Technologien kann das Verhältnis demnach positiv beeinflussen. Darüber hinaus wurde eine Arbeit publiziert, in der theoretische Grundlagen für eine später geplante Studie dargestellt wurden. In dieser soll systematisch die Beziehung von Patienten zu neuen mobilen Technologien in Hörgeräten untersucht werden. Es wurden bisher nur Kriterien für die Literaturrecherche, sowie Ein- sowie Ausschlusskriterien definiert und publiziert [66]. Leider waren bis zur Abgabe des vorliegenden Manuskriptes noch keine weiteren Daten und Ergebnisse dazu veröffentlicht.

\subsubsection{Studien zu Apps zum Thema Kopf-Hals-Tumoren}

Drei Studien zum Thema Apps und Kopf-Hals-Tumoren wurden recherchiert [72-74]. In einer Arbeit wurde in einer Kohorte von Patienten, in der auch Betroffene mit Kopf-Hals-Tumoren eingeschlossen waren, unter Zuhilfenahme eines Fragebogens evaluiert, ob sie sich vorstellen könnten, dass die Tumortherapie aber auch die Lebensqualität mit Hilfe einer App überwacht und ggf. gesteuert werden könnten [72]. Der Auswertung zufolge halten es vorwiegend jüngere Patienten sinnvoll, Daten über eine App einzupflegen um davon profitieren zu können. Kritische Punkte waren aber die Sicherheit der eingegebenen Daten und die komplizierte Anwendung. In einer weiteren Studie wurde eine App eingesetzt, die dazu dienen soll die individuelle Lebensqualität von Patienten mit Kopf-Hals-Tumoren zu kontrollieren und durch spezielle Empfehlungen zu verbessern. [74]. Nach Angaben der Autoren war die Teilnehmer mit dem Produkt zufrieden. Daher soll nun die App weiter verbessert werden um eine nachhaltige Verbesserung der Lebensqualität zu erreichen. Die Früherkennung von Kopf-Hals-Tumoren sowie das Verbessern einer solchen mit Hilfe einer App war Gegenstand einer anderen Studie [73]. In dieser App werden per Fragenbogen Risikofaktoren für die Entstehung von Kopf-Hals-Tumoren, wie z. B. Noxen oder ungeschützter Oralverkehr, abgefragt. Die App erlaubte auch Bilder der betroffenen Schleimhaut aufzunehmen und durch erfahrene Diagnostiker im Kopf-Hals-Bereich auswerten zu lassen und wurde bei Risikopatienten für die Entstehung von Kopf-Hals-Tumoren getestet. Spezifität und Sensitivität waren nicht so hoch wie bei der direkten klinischen Anamnese und Untersuchung. Dennoch sind die Autoren überzeugt, dass eine solche die App bei der Früherkennung von Malignomen hilfreich sein kann.

\subsubsection{Studien zu Apps zum Thema Allergologie}

Zu diesem Bereich wurden insgesamt 6 Studien gefunden [75-80]. In einer Arbeit wurden die theoretischen Grundlagen und die mögliche Entwicklung einer App im Bereich der allergischen Rhinitis beschrieben [79]. Betroffene könnten damit ein Screening für das Vorliegen einer allergischen Rhinitis durchführen. Für Ärzte stehen wei- 
tere Arbeitsinstrumente zur Verfügung z. B. die digitale Erhebung von Symptomen über eine visuelle Analog-Skala. In einem Positionspapier, an dem die HNO-Fachgesellschaft beteiligt war, wurde die digitale Erhebung von Symptomen der allergischen Rhinitis über eine App als sinnvoll beschrieben [75]. Entsprechend wurde dies in einer Folgestudie validiert [76]. In einer weiteren Publikation wurde gezeigt, dass die über eine solche App erhobenen Beschwerden mit dem Arbeitsausfall von Betroffenen korrelierten, was die Funktion und Genauigkeit der App unterstreicht [78]. Über diese App konnten an einer Kohorte von Patienten auch die allergischen Symptome unter Medikation kontrolliert werden [77]. Über eine gerichtete Hyposensibilisierung wurde in einem Case Report mit 2 Patienten mit allergischer Rhinitis berichtet, die eine überlappende Sensibilisierung von Allergenen aufwiesen. Die in der App täglich aufgezeichneten Symptome wurden mit dem Pollenflugkalender in Korrelation gesetzt und dann die Allergene mit der höchsten Sensibilisierung eruiert sowie die Patienten gegen diese hyposensibilisiert [80].

\subsubsection{Studien zum Thema „Hearables“}

Hearables sind Kopfhörer, die mit zusätzlichen Funktionen wie z. B. eine Drahtlosverbindung zu einem Smartphone oder mit Sensoren zur medizinischen Überwachung ausgestattet sind. 3 Studien wurden dazu gefunden [81-83]. In einer Arbeit wurde die Möglichkeit untersucht mit einem solche System neuronale auditorisch evozierte Potentiale im Gehörgang abzuleiten [81] mit dem Ziel, die Einstellmöglichkeiten von Hörgeräten insbesondere in schwierigen Hörsituationen zu verbessern. Es wurden Hearables mit mehreren Elektroden entwickelt, mit denen man über direkten Kontakt mit der Gehörgangshaut reproduzierbare Potentiale ableiten konnte. Die Sensitivität hängt dabei - nach Aussage der Autoren - direkt von der Lage der Kontakte ab. In 2 weiteren Studien wurden physiologische Parameter, wie z. B. die Herzfrequenz, das EEG und auch sprachsynchrone Atembewegungen mit speziell entwickelten Hearables abgeleitet $[82,83]$. Es waren jedoch sehr komplexe Signalverarbeitungsstrategien erforderlich um die Signale zu identifizieren

\subsubsection{Studien zu Apps in sonstigen Bereichen}

Unter „Sonstige“ fallen Themenbereiche, zu denen jeweils nur eine Studie gefunden wurde. [84-87]. Bei einer dieser Arbeiten handelte sich um eine App, mit der es möglich ist eine Rhinoplastik zu planen [87]. Die Funktion der App und ihre Einsetzbarkeit wurden durch erfahrene Chirurgen evaluiert und mit dem Goldstandard, der auf Fotos basierten OP-Planung, verglichen. Mithilfe der App konnte die präoperative OP-Planung erleichtert und die Darstellung der Operation für den Patienten vereinfacht werden. Eine weitere App enthielt vorgefertigte Zeichen und Videos in der Gebärdensprache [84]. In einer Publikation wurde ein Überblick über 9 Apps erstellt, die im Rahmen einer gastroösophagealen Refluxerkrankung eingesetzt werden können. [85]. Die meisten dieser Apps weisen nach Angaben der Autoren jedoch deutliche Mängel in ihrer Funktion und/oder der Benutzerfreundlichkeit auf. Für den Bereich der Cochlea-Implantate gibt es eine Arbeit zu einer App, mit der die Einstellung des Implantates selbst durch den Nutzer verändert werden kann [86]. Erste Untersuchungen zeigten, dass die Cl-Träger im Stande waren die Einstellungen an ihrem Gerät selbst zu verändern. Obwohl sich das durch den Träger erstellte individuelle Programm deutlich von der vorherigen
Einstellung unterschied, konnte dennoch ein vergleichbares Sprachverständnis erreicht werden.

\subsection{Fazit}

Im Rahmen der Literaturrecherche konnten viele Studien gefunden werden, die Apps im Bereich der HNO-Heilkunde zum Thema hatten. Es fand sich seit dem Jahr 2013, in dem die erste Studie zur Thematik veröffentlicht wurde, eine deutlich zunehmende Anzahl an Publikationen. Dies zeigt, dass sich der Fachbereich der HNO-Heilkunde zunehmend mit dieser Thematik wissenschaftlich auseinandersetzt. Die meisten Studien waren aber beschreibenden Charakters und/oder setzten keine Kontrollgruppe ein, so dass belastbare Aussagen zu den Vorzügen der App-basierten Ansätze kaum möglich sind. Dies unterstreicht die Notwendigkeit, Standards zu generieren, wie Apps in Zukunft wissenschaftlich untersucht werden können. Dies könnte dann als Voraussetzung für eine weitere Zertifizierung dienen, um die App ohne rechtliche Bedenken im Bereich der Patientenversorgung, der Forschung, aber auch der Administration einsetzen zu können.

\section{Umfrage zu „Apps in der HNO-Heilkunde“}

Die Bewertung einer App, insbesondere für den Einsatz im klinischen Alltag, ist komplex, da keine Standards vorliegen und viele Parameter zu beachten sind. Von den Herstellern werden die Apps dem potentiellen Nutzer angepriesen ohne dabei die Risiken zu erwähnen. Ohne eine objektive und standardisierte Bewertung kann der Einsatz in der Patientenversorgung, aber auch die Nutzung in klinischen Studien zu erheblichen, auch rechtlichen Problemen führen. Apps sollten daher nach einem standardisierten Bewertungsmaßstab evaluiert werden. Damit ließe sich zumindest einigermaßen objektiv beurteilen, ob das Produkt einen Einsatz in der klinischen Routine finden kann. Ein solcher Maßstab wurde im Jahre 2013 als „App-Synopsis“ veröffentlicht [88]. Ziel war es mobile medizinische Apps transparent beurteilen zu können. Die Bewertung nach der „AppSynopsis“ sollte dann bei den angebotenen Apps als zusätzliche Beschreibung in den Stores erscheinen [88]. Hierzu sind zu den Kategorien „Impressum“, „Rationale“, „Funktionalität“, „Validität und Zuverlässigkeit“ sowie „Informationsabfrage und Verwaltung“ Daten anzugeben. Zusätzlich gibt es weitere Unterpunkte wie z. B. Fragen nach dem Hersteller und dem Sponsoring, aber auch zur Datenverarbeitung und -speicherung [89]. Beispielhaft sei hier die öffentlich zugängliche Bewertung nach der „App-Synopsis“ für die App „iSignIT“ genannt [84]. Darüber hinaus wurde auch eine „App-Synopsis für Nutzer" entwickelt, die es Anwendern ermöglicht eine medizinische App nach definierten Kriterien zu evaluieren [90]. Diese Checkliste für Nutzer enthält insgesamt 39 Fragen, die mit „ja“, „,nein“ oder „unklar“ beantwortet werden können. Die Fragen sind den Kategorien „Medizinprodukt“, „Zweckbeschreibung“, „Funktionalität“, „Wissenschaftlichkeit“, „Einschränkungen und Limits“, „Risiken“, „Verlässlichkeit und Inhalte“, „Datenschutz“ und „Impressum“ zugeordnet. Die möglichen Antworten sind in den Ampelfarben markiert, so dass der Nutzer schon beim Ankreuzen das Risiko des Ein- 
satzes der App einfacher beurteilen kann (rot = hohes Risiko/ grün = niedriges Risiko).

\subsection{Konzept der Umfrage}

Um im Rahmen des Referates einen Überblick zu erstellen, welche Apps im klinischen Alltag im Fachgebiet der HNO-Heilkunde zum Einsatz kommen können, wurde eine eigene Studie an der Universitäts-HNO-Klinik Würzburg initiiert. Hierzu erging Anfang Mai 2018 ein Aufruf an alle Mitarbeiter der Klinik, eine frei zu wählende App nach der oben beschriebenen „App Synopsis für Nutzer“ zu bewerten. Der Fragebogen wurde mit Fragen nach dem Teilgebiet, „Hals“, „Nasen“ oder „Ohr“ bzw. „Sonstige“ ergänzt. Darüber hinaus wurde nach dem Einsatz in der Klinik oder in der Forschung gefragt und um eine kurze Beschreibung als Freitext gebeten. Es wurde ein Onlinefragebogen entworfen und der entsprechende Zugriffslink an die Mitarbeiter per Email versandt. Die Antworten wurden bis zum 30.06.2018 gesammelt.

\section{2 Überblick über die bewerteten Apps}

Insgesamt wurden 33 Apps genannt. 23 Apps wurden auf dem Betriebssystem Apple iOS und 10 Apps auf Google Android getestet. 15 Apps wurden dem Teilgebiet „Ohr“, 6 Apps dem Teilgebiet „Nase“, 10 Apps dem Teilgebiet „Sonstige“ und 1 App dem Teilgebiet „Hals“ zugeordnet. Die evaluierten Apps konnten zu 14 Gruppen zusammengefasst werden. Es wurden 4 Apps bewertet, die im Bereich der Allergologie eine digitale Vorhersage des Pollenfluges ermöglichen. Zwei Apps wurden untersucht, die bei der Kommunikation in Gebärdensprache Hilfestellung bieten. Im Bereich der Neurootologie wurden 3 Apps getestet, die Anleitungen zur Diagnostik und/oder Therapie von Gleichgewichtsfunktionsstörungen zum Thema hatten. Drei Apps zu Hörtests und 4 zum Hörtraining nach Cochlea-Implantation wurden ebenso bewertet wie ein System zur Schallpegelmessung mit dem Smartphone. Zwei Apps waren Anwendungen zur korrekten Medikamentendosierung. Vier Apps umfassten verschiedene Nachschlage-Funktionen für das Fach der HNOHeilkunde und 2 Apps digitale Operationslehren. Darüber hinaus wurde 1 App zur Rauchentwöhnung, 2 Apps aus dem Bereich der Schlafmedizin mit Monitoringfunktion des Schlafes, 1 App im Bereich Phoniatrie/Stimme und 2 Apps zur Tinnitustherapie analysiert. In \ Abb. $\mathbf{3}$ ist die Verteilung der einzelnen Themenbereiche dargestellt, > Tab. 3 enthält eine ausführliche Beschreibung der Funktionen der bewerteten Apps.

\subsection{Auswertung des Fragebogens}

Das Erkennen eines möglichen Risikos beim Einsatz einer App war ein wichtiger Punkt bei der Auswertung der Umfrage. Daher wurden primär die Fragen und Antworten ausgewertet, bei der die Angabe „nein“ die Markierung eines roten Feldes zur Folge hatte. Dies soll dem Nutzer bereits als Signal dienen, dass die Anwendungssoftware an diesem Punkt eine deutliche Schwachstelle aufweist. Die erste Frage, bei der eine Beantwortung mit „nein“ zu der Markierung eines roten Feldes führt, ist „Wird der Zweck der App verständlich beschrieben?“. Diese Frage wurde bei 4 Apps mit „nein“, beantwortet. Bei der Frage „Werden die Funktionen und Leistungsmerkmale verständlich und plausibel dargestellt?“ wurde bei 3 Apps mit „nein“ geantwortet. Die Frage „Wird plausibel erläutert, dass die Entwicklung auf

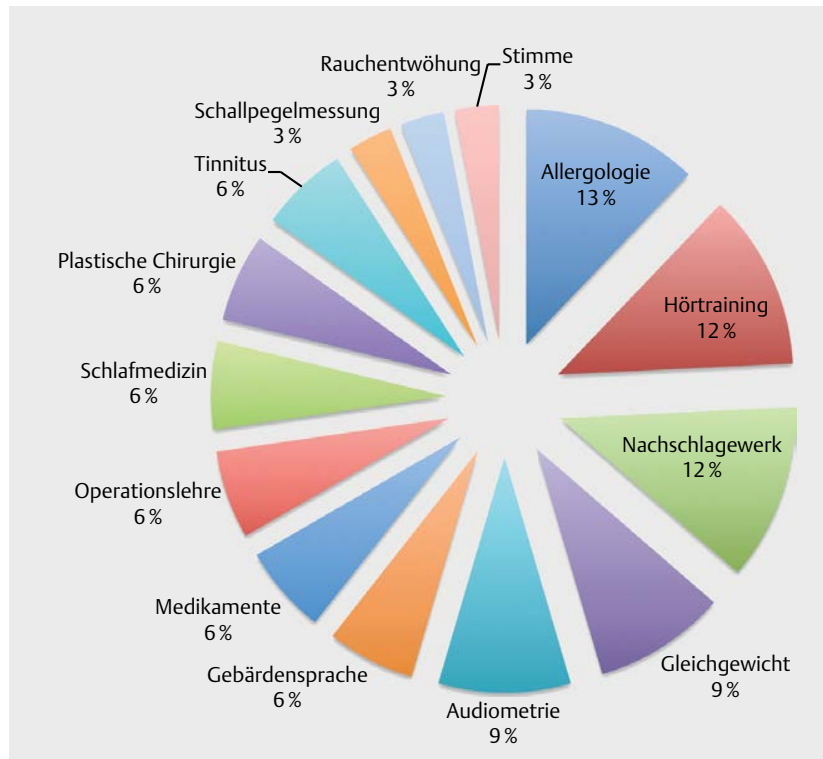

- Abb. 3 Prozentuale Verteilung der mit der „App Synopsis für Nutzer“ bewerteten Apps in der HNO-Heilkunde.

dem aktuellen Stand von Wissenschaft und Technik beruht? “ wurde in 13 Fällen mit „nein“ beantwortet. Auch bei der Frage „Werden Informationen über die Autoren der Inhalte gemacht, inkl. ihrer Namen und Qualifikationen?“ war in 13 Fällen die Antwort „nein“. Ein „nein“ ergab sich bei der Frage „Werden die Quellen der Inhalte angegeben und sind diese seriös? “ in 17 Fällen, bei der Frage "Können Sie die gewünschten Funktionen der App auch ohne Dateneingabe nutzen? Ist die Dateneingabe freiwillig?“ in 6 Fällen und bei der Frage „Haben Sie Möglichkeiten, die Datenerfassung oder den Datenversand zu steuern und wird Ihnen mitgeteilt, wie Sie diese Einstellungen vornehmen können?" in 14 Fällen.

Nach den erläuterten Regeln (s. o.) bedeutet die Beantwortung aller Fragen mit „ja“ eine hohe Sicherheit bei der Anwendung. Die Auswertung ergab, dass nur bei 4 Apps (12\%) alle Fragen mit ,ja“ beantwortet wurden. Toleriert man ein zusätzliches „nein“ und schließt damit 2 Apps (6\%) mit ein, ergibt sich bei lediglich 18\% der Apps eine ausreichende Sicherheit beim Einsatz. Im Fragebogen gibt es 2 weitere Fragen von besonderer Relevanz, deren Beantwortung mit ,ja“ zu einer Markierung eines grünen Feldes führt, also zu einer Empfehlung des Einsatzes. Dies ist die Frage „Handelt es sich um ein Medizinprodukt (Trägt es eine CE-Kennzeichnung oder hat es eine FDAZulassung)?“. Diese Frage wurde in 2 Fällen mit „ja „, beantwortet. Auch die Frage „Werden wissenschaftliche Studien zur App angegeben und sind diese seriös?“ wurde nur in 2 Fällen mit „ja“ beantwortet. Die Auswertung beider Fragen ergab, dass nur eine der bewerteten Apps bei beiden Fragen die Bewertung „ja“ erhielt. Betrachtet man die Kombination aus Positiv- und Negativfragen ergab sich, dass keine der bewerteten Apps eine Bewertung mit „ja“ bei Positivfragen und keine Bewertung mit „nein“ bei den Negativfragen hatte.

\subsection{Fazit}

Insgesamt ergab die Bewertung der Apps nach der „App-Synopsis für Nutzer“, dass keine der bewerteten Apps uneingeschränkt für 


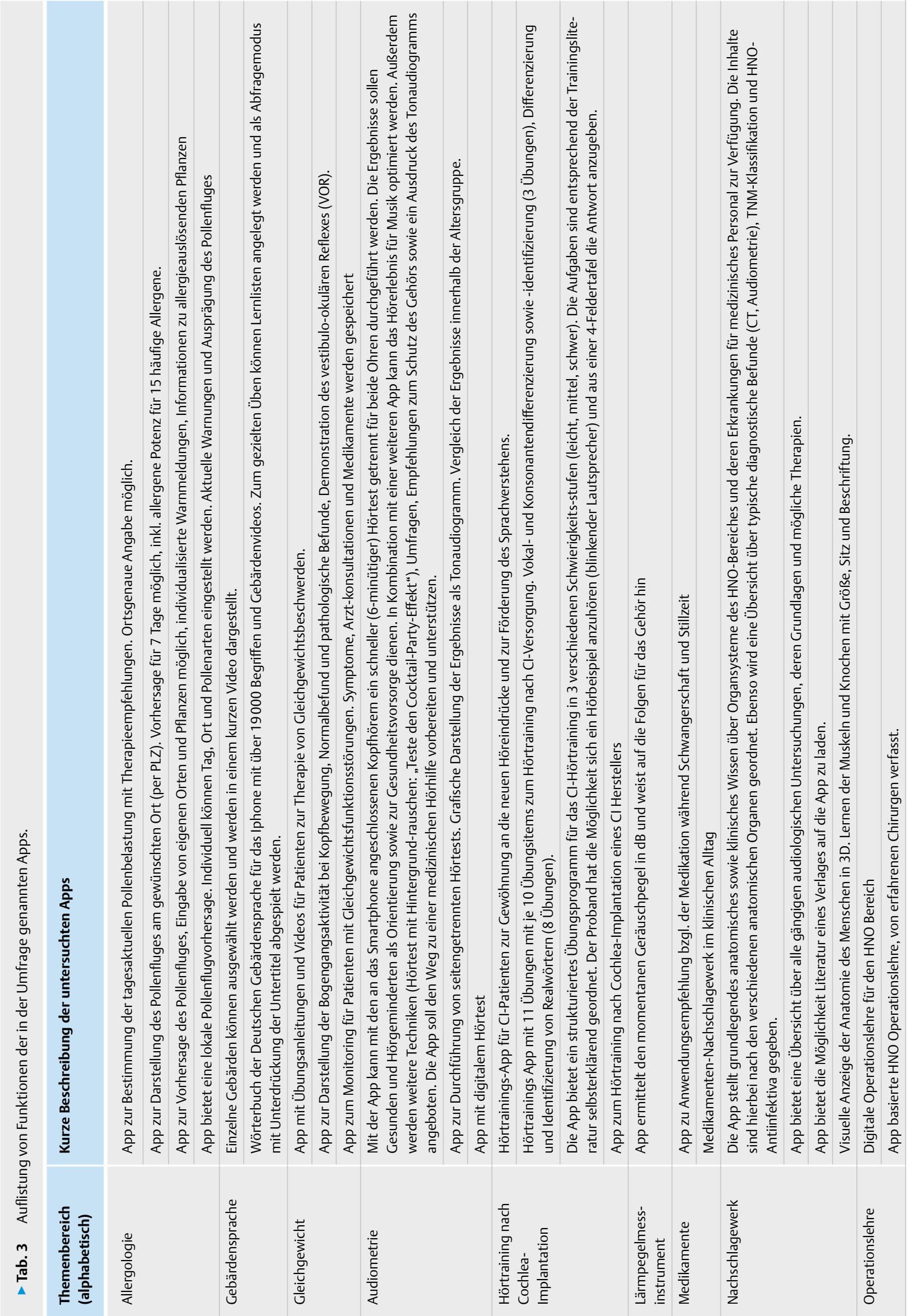


den Einsatz empfohlen werden kann. Leider werden im Bereich der HNO-Heilkunde, wie in anderen medizinischen Bereichen auch, die Möglichkeiten Apps zu zertifizieren (Medizinprodukt oder CE-Kennzeichnung) kaum eingesetzt. Häufig gibt es keine ausreichenden Angaben zu dem aktuellen Stand von Wissenschaft und Technik, was bei einer medizinischen App Standard sein sollte. Aber auch die fehlende Transparenz im Hinblick auf Einschränkungen und Limitierungen sowie fehlende Informationen zu datenschutzrechtlichen und von der Nutzung der App ausgehende Risiken wurden bemängelt. Der Einsatz des strukturierten und systematischen Bewertungsmaßstabes zur Begutachtung von medizinischen Apps führt in jedem Fall dazu, dass mit diesem solche Anwendungen mit einem geringen zeitlichen Aufwand evaluiert werden können. So erhält der Nutzer die notwendigen Informationen sowohl über die Schwachstellen, wie auch die positiven Aspekte der Anwendungssoftware. Jeder Nutzer sollte somit nach der Bewertung prüfen, ob der Nutzen des Einsatzes der App die Risiken aufwiegt.

\section{Entwicklung einer App zum Training der mimischen Muskulatur bei Fazialisparese}

Nach eingehender Auseinandersetzung mit den auf dem Markt befindlichen Apps mit Bezug zur HNO-Heilkunde war es das Ziel, eine für den Nutzer bzgl. Funktionalität und Verlässlichkeit der Inhalte einwandfreie und transparente App zu entwickeln. Einschränkungen und Limitierungen sowie auch Risiken sollten im Rahmen der Entwicklung beachtet werden. Somit sollte eine Anwendung, welche die Kriterien einer guten und qualitativ hochwertigen App erfüllt, erstellt werden (siehe Absatz 3 f). Dies schließt alle rechtlichen Vorgaben in Bezug auf die Datenverarbeitung und -speicherung ein, auch sollten die wesentlichen Aspekte einer Zertifizierung als Medizinprodukt mit Eingang finden.

\subsection{Konzeptionierung}

Bei der Konzeptionierung wurden zunächst mehrere Themenbereiche und Umsetzungsformen in Betracht gezogen. Selbstverständlich sollte die App inhaltlich neuartig, mit überschaubarem Aufwand umsetzbar, einfach bedienbar und mit klinischem Nutzen verbunden sein. Allgemein gilt es beim Entwicklungsprozess u. a. zu klären, ob eine native, hybride oder Web-basierte App-Architektur gewählt werden soll. Eine App wird als nativ bezeichnet, wenn diese für ein spezifisches Betriebssystem des jeweiligen mobilen Endgeräts mit einer entsprechenden Programmiersprache und Entwicklungsumgebung produziert wird. Eine Web-basierte App hingegen ist eine Website, die für die Anzeige auf mobilen Endgeräten optimiert ist, aber auch mit komplexen Funktionalitäten ausgestattet werden kann. Wesentliche Vorteile dieses Vorgehens sind die relativ schnelle und kosteneffiziente Umsetzung sowie die Plattform-Unabhängigkeit. So können Web-basierte Apps problemlos sowohl z. B. für iOS- als auch für Android-Geräte zur Verfügung gestellt werden, letztlich auch ohne den „Umweg“ über den Apple AppStore bzw. Google Play Store zur Distribution nehmen zu müssen. Als Nachteile von Web-basierten Anwendungen sind die teils deutlich eingeschränkten Nutzungsmöglichkeiten von Hard- und Software der Geräte, wie z. B. Kamera, Mikrofon, Ortungsfunktion und Sicherheitsfeatures, zu nennen. Zudem ist der Nutzer in der Regel auf eine 
Internetverbindung angewiesen. Hybrid-Apps schließlich basieren, so wie auch Websites, auf einfachen Skriptsprachen (HTML, CSS und Javascript) und werden über ein entsprechendes Framework als eine Art Container in eine spezifische mobile Architektur (z. B. iOS oder Android) implementiert. Nach außen hin erscheint eine Hybrid-App also wie eine native App, kann auf viele Hard- und Softwarefeatures der Endgeräte zugreifen und ist offline nutzbar. Nachteilig jedoch ist unter anderem die geringere Leistungsfähigkeit.

\subsection{Auswahl des Themas}

Da die Klinik-Website der HNO- Klinik am Uniklinikum Würzburg schon für die jeweils optimale Anzeige auf Desktoprechnern, TabletPCs und Smartphones ausgerichtet ist, wurde die Idee verworfen eine (Hybrid-) App zur Außendarstellung der Klinik zu entwickeln. Als geeignete Aufgabenstellung für eine neue App ergab sich ein klinisches Thema: eine spezielle Anleitung zu einem gezielten Training der mimischen Muskulatur bei Patienten mit einer Fazialisparese. Im klinischen Kontext war hier ein Optimierungsbedarf gesichtet worden. Fazialistraining als nicht-invasiver Therapieansatz wird Patienten mit Fazialisparese unterschiedlicher Genese empfohlen und umfasst funktionelle motorische Übungen zur Reanimation der mimischen Muskulatur im Sinne eines neuromuskulären Trainings. Die aktuelle Studienlage zeigt, dass mit einer strukturierten Therapie im Rahmen eines interdisziplinären Konzepts gute Therapieerfolge erzielt werden können und diese zur Vermeidung von Synkinesien oder Hyperkinesien beitragen kann [91-94]. Bisherige verfügbare Übungsbögen weisen einen Optimierungsbedarf auf, die visuelle Kontrolle unter Nutzung eines Spiegels ist erfahrungsgemäß mit einer geringen Compliance verbunden.

Aus dieser Situation heraus entstand die Idee, eine App für strukturierte Fazialisübungen mit visuellem Feedback über die Frontkamera des Smartphones zu entwickeln. Eine Recherche im Apple AppStore und im Google Play Store, sowie eine Literaturrecherche via PubMed ergab, dass bislang keine adäquate App mit der genannten Zielsetzung existiert. Einzig eine kostenpflichtige und englischsprachige App bietet Fazialisübungen an, jedoch nur in Form eines digitalen Übungsbogens ohne visuelles Feedback [95]. Zur optimalen Nutzung der Gerätehardware fiel die Entscheidung auf eine native App-Architektur unter iOS, welche als besonders sicher und benutzerfreundlich angesehen werden kann. Die Umsetzung für Android-Geräte im Verlauf ist geplant.

\subsection{Struktur und Aufbau der App}

Es wurden verschiedene Grundfunktionen der App erarbeitet. Zur Einführung in die Thematik sollten für den Patienten verständliche Erklärungen über Anatomie und (Patho-) Physiologie des Nervus facialis sowie über die Funktionsweise der App enthalten sein. Als zentraler Punkt der App war ein Trainingsmodus mit schrittweisen Anleitungen zu mimischen Übungen mit visuellem Feedback über die Frontkamera angedacht. Dabei sollen die Anweisungen als Text und zur besseren Verständlichkeit auch über ein animiertes „Smiley“-System angeboten werden. Eine Timerfunktion soll den Trainingsfortschritt abbilden. Nach abgeschlossenem Training soll eine Erfolgsmeldung zur positiven Verstärkung beitragen. Durch (optionale) Trainings-Erinnerungen über das iOS Notification Center könnte eine Erhöhung der längerfristigen Compliance erzielt werden. Darüber hinaus ist ein Impressum mit entsprechenden Hinweisen zu Haf- tungsbestimmungen und zum Datenschutz gemäß DatenschutzGrundverordnung obligat.

Als künftige Ergänzung ist eine Erfassung des Lähmungsgrades durch die Anwendung eines definierten Scores angedacht. Diese könnte zunächst manuell nach einem oder mehreren gängigen Scoresystemen (Stennert-Index, House-Brackman-Skala, Facial Grading System) erfolgen, theoretisch wäre aber auch der Einsatz der Gesichtserkennungsfunktion über die TrueDepth Kamera des aktuellen bzw. künftiger iPhones denkbar. So konnten Pourmomeny et al. zeigen, dass ein softwarebasiertes Gradingssystem bei Fazialisparesen zuverlässige Ergebnisse liefert [96]. Die somit erhobenen Score-Werte könnten in einer Verlaufsgrafik abgebildet werden. Um einem Missbrauch der Daten vorzubeugen, sollen die Daten ausschließlich auf dem jeweiligen Endgerät gespeichert werden.

\subsection{Grafische Gestaltung und Programmierung der App}

Die grafische Konzeptionierung und das Layout erfolgten mit Adobe Photoshop, Adobe Illustrator und der Sketch App. Während die genannten Adobe-Produkte Industriestandard zur pixel- bzw. vektorbasierten Bildbearbeitung sind, ist Sketch insbesondere für das UserInterface-Design mobiler Endgeräte optimiert und ermöglicht somit eine erste Simulation der künftigen App. Bei der Konzeptionierung wurden die Vorgaben des Corporate Designs des Uniklinikum Würzburg eingehalten. Die Animationen der Gesichtsbewegungen zum mimischen Training wurden in Adobe After Effects erstellt und mit dem Bodymovin-Plugin exportiert, um schließlich über das OpenSource-Framework Lottie in das Xcode-Projekt integriert zu werden. Dieses und weitere Frameworks wurden mit dem Dependency Manager Cocoapods in das Projekt integriert. Die Programmierung per Swift bzw. Objective-C und die eigentliche Erstellung der grafischen Benutzeroberfläche erfolgte mit der Entwicklungsumgebung Apple Xcode. Mithilfe dieser Software lassen sich die einzelnen Seiten gestalten und deren Bedienelemente mit dem Programmcode verknüpfen. Ein hilfreiches Feature von Xcode ist, dass während des Entwicklungsprozesses die App sowohl auf virtuellen als auch physischen Endgeräten mehrerer Generationen getestet und optimiert werden kann. ( Abb. 4).

\subsection{Weitere Entwicklung und Validierung}

Nach erfolgreicher Entwicklung der App ist geplant diese im Rahmen einer klinischen Studie zu evaluieren. Hierzu könnte man sich der Möglichkeiten des Apple Research Kits bedienen, welches sehr attraktive Möglichkeiten zur schnellen und großflächigen Datenerhebung ohne Medienbruch bietet und bereits von weltweit führenden medizinischen Instituten eingesetzt wird [97]. Zusätzlich soll die App auch nach dem objektiven Bewertungsmaßstab für medizinische Apps der „App-Synopsis“ beschrieben [88] und auch nach der „AppSynopsis für Nutzer“ nach definierten Kriterien evaluiert werden [90].

\subsection{Fazit}

Im Rahmen des Entwicklungsprozesses der App zum Training der mimischen Muskulatur nach Facialisparese war es möglich, eine Anwendungssoftware zu konzeptionieren und erste Versionen zu programmieren, welche das Potenzial hat, die Kriterien einer guten und 


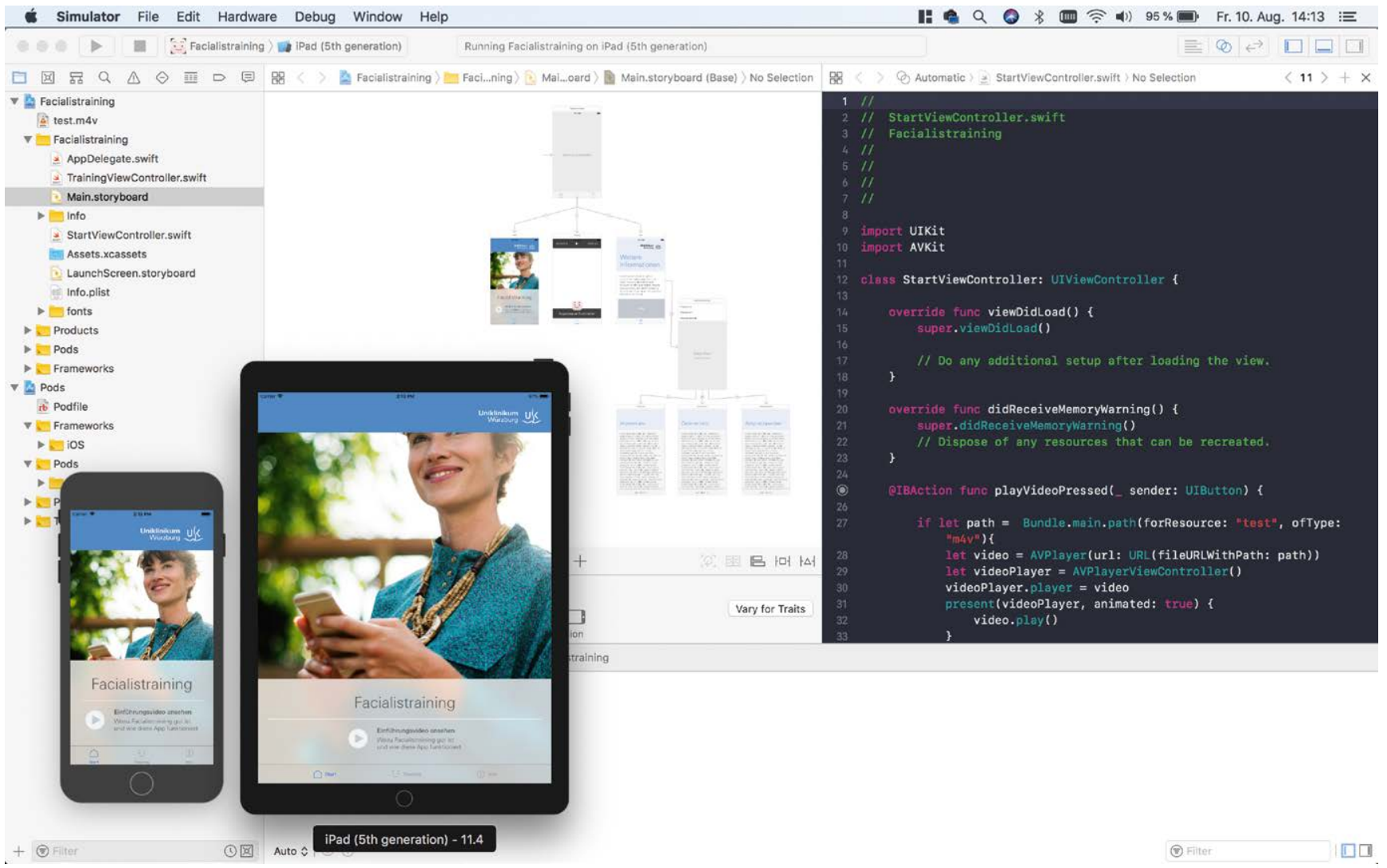

- Abb. 4 Grafische Konzeptionierung, Layout und Simulation im Programm „Sketch App“.

qualitativ hochwertigen App zu erfüllen. Die weitere Entwicklung und Validierung der App wird zeigen, ob die App funktionell ist und einen Benefit für den Patienten hat. Diese Erkenntnisse werden die Grundlage für den weiteren Entwicklungsprozess, aber auch für eine mögliche Zertifizierung der App als Medizinprodukt sein.

\section{Zusammenfassung und Ausblick}

Die Nutzung mobiler Informations- und Kommunikationstechnologie in der Medizin, wozu auch medizinische Apps zählen, wird als "Mobile Health" bezeichnet. Dieser Bereich gewinnt immer mehr an Bedeutung, da er neue Lösungsansätze im Gesundheitswesen bietet. Das Bewusstsein, dass Digitalisierung auch in der Medizin ihren Platz finden muss, hat in der Ärzteschaft in den letzten Jahren deutlich zugenommen, was sich auch an der Etablierung von Arbeitsgruppen bzw. Task Forces diverser berufsständiger Organisationen zeigt. Der Einsatz dieser Technologie bietet große Vorteile, wie z. B. die direkte Verfügbarkeit oder die Unabhängigkeit von Öffnungszeiten, was zu einer Flexibilität des Einsatzes im Alltag führen kann. Die neue Technologie birgt aber auch Risiken, wie z. B. eine ungefilterte Datenspeicherung, die zu einer Beeinträchtigung von Persönlichkeitsinteressen führen kann. Schon heute wird das Arzt-Patient-Verhältnis durch diese neuen Technologien beeinflusst, was eine ausführliche und sorgfältige Auseinandersetzung mit diesem Thema unabdingbar macht.

Auf dem Markt findet sich eine immer größere Anzahl von medizinischen Apps, die auch den HNO-Bereich betreffen. Die Anzahl von deutschsprachigen Anwendungen, die unserem Fachgebiet zugeordnet werden können, ist mit ca. 2 \% noch sehr klein, wobei die Apps im Vergleich zur gesamten Gruppe der medizinischen Apps häufiger mit Updates versorgt, von der Dateimenge größer und auch sorgfältiger beschrieben sind. Dies kann auf ein höheres Verantwortungsbewusstsein der Hersteller und eine bessere Qualität der Apps hinweisen. Jedoch ist auch eine prozentual höhere Anzahl von Apps kostenpflichtig, wobei nur für wenige Apps eine Kostenübernahme durch die Krankenkassen angegeben wird.

Medizinische Apps können Medizinprodukte sein oder deren Funktion übernehmen. Dies führt zu einer besonderen rechtlichen Stellung, insbesondere im Schadensersatzfall. Der Nutzer kann nicht davon ausgehen, dass eine App ein Medizinprodukt ist, auch wenn sie für medizinische Zwecke eingesetzt werden kann. Der Hersteller legt fest, ob er seine Anwendung als Medizinprodukt deklarieren möchte, was dann mit einem sehr aufwändigen Zulassungsverfahren behaftet ist. Daher wählen die meisten Anbieter nicht die aufwändige Zertifizierung als Medizinprodukt, da die Apps dann grundlegende Anforderungen nach Dokumentation, Softwareentwicklung, Tests, Validierung, Gebrauchstauglichkeit, Risikomanagement und Versionsmanagement erfüllen müssen. Anderseits sind sich die Anbieter häufig dieser Problematik auch gar nicht bewusst. Für den Arzt führt das zu einer besonderen Verantwortung, da er dem Patienten eine nach allgemein akzeptierten fachlichen Standards anerkannte Behandlung schuldet. Empfiehlt er nun eine App, die nicht als Medizinprodukt gekennzeichnet ist, kann er theoretisch aufgrund 
des Einsatzes ungeeigneter Instrumente in Haftung genommen werden. Dies trifft auch zu, wenn die App abweichend von ihrer Zweckbestimmung eingesetzt wurde. Somit ist vom Arzt eine hohe Sorgfalt bei der Auswahl und der Empfehlung einer App zu erwarten, um haftungspräventiv zu handeln. Auch andere Qualitätssiegel sind selten. Eine staatliche Prüfung der Anwendungssoftware kann schon aufgrund der großen Zahl der in den Stores befindlichen Apps nicht stattfinden. Ebenso ist, bedingt durch die mangelnde Transparenz, selten ersichtlich, welche Bewertungsmaßstäbe private Zertifizierungsanbieter einsetzen. Vermehrt versuchen auch branchenferne Hersteller im Bereich der medizinischen Apps Fuß zu fassen, ohne genügend Erfahrung und Expertise in Bezug auf die medizinische Thematik und unter Vernachlässigung branchenüblicher Standards und sogar regulatorischer Vorgaben. Manchmal überschätzen die Entwickler der App auch die technischen Möglichkeiten und wiegen so den Nutzer in einer trügerische Sicherheit bzw. verleiten ihn zu einer möglicherweise gefährlichen Fehleinschätzung seines Gesundheitszustandes.

Zur Wirksamkeit von medizinischen Apps fehlt meist bisher die wissenschaftliche Evidenz, was sich auch im geringen Erstattungskontext der Krankenkassen widerspiegelt. Nützt man medizinische Apps trotzdem, so wäre es wünschenswert, dass die Konzeptionierung und Ausführung der Anwendung gut gestaltet ist Eine solche würde sich dadurch auszeichnen, dass das Nutzungserlebnis dem Anwender zu jedem Zeitpunkt die Gewissheit gibt, mit einer sorgfältig entwickelten Anwendung zu arbeiten, die eine Gefährdung der Gesundheit wie auch seiner sozialen und wirtschaftlichen Situation ebenso wie Risiken für seine Umwelt von vornherein ausschließt. Der Einsatz einer so gestalteten App kann zu einer hohen Zufriedenheit des Nutzers führen und eine angemessene Wahrscheinlichkeit auf einen Therapieerfolg beinhalten. Dies steht im Gegensatz zu einer schlecht konzeptionierten und ausgeführten App, bei der eine geringe Akzeptanz und kein bleibender Erfolg zu erwarten sind.

In der wissenschaftlichen Literatur finden sich zwar zahlreiche Veröffentlichungen zu medizinischen Apps, jedoch sind die meisten auf eng gesteckte Anwendungsbereiche fokussiert, sodass eine Übertragung auf den gesamten Sektor und generelle Schlussfolgerungen daraus unwissenschaftlich wären. Jedoch konnte gezeigt werden, dass einige Funktionen der Smartphones, wie z. B. die Erinnerungsfunktionen oder das Unabhängigkeitskonzept, zu einer positiven Bewertung solcher Apps und damit zu einer verbesserten Partizipation an Gesundheitsprozessen führen können. In einer Literaturrecherche für das vorliegende Referat konnten mehr als 70 Studien identifiziert werden, die verschiedenen Teilbereichen des Fachgebietes HNO-Heilkunde zuzuordnen waren. Dieses waren im Speziellen die Themen „Audiometrie“, "Schallpegelmessgerät“, „Edukation“, „Tinnitus“, „endo- und otoskopische Bildgebung“, „Schlafmedizin“, „Stimme“, „Gleichgewicht“, „Schlucken“, „Hörgerät“, „,Kopf-Hals-Tumoren“, „Allergologie“, „Hearables“ und „Sonstige“. In der Mehrzahl der Publikationen wurden lediglich die neue Funktion bzw. die App beschrieben und es fehlte an Kontrollexperimenten, so dass die wissenschaftliche Qualität dieser Arbeiten als deutlich eingeschränkt zu bewerten ist. Dennoch ermöglichte diese Literaturrecherche einen Überblick zur vorhandenen Einsatzbreite von Smartphones und Apps in der HNO-Heilkunde.

Die für das Referat an der Univ. HNO Klinik Würzburg durchgeführte Umfrage ergab einen breiten Einsatzbereich von Apps im
Fachgebiet der HNO-Heilkunde. Es wurden insgesamt 33 Apps benannt, die einzelnen Teilgebieten der Faches bzw. Thematiken zugeordnet werden konnten. Diese enthielten die Bereiche „Allergologie“, „Gebärdensprache“, „Gleichgewicht, „Hörtest“, „Hörtraining nach Cochlea-Implantation“, „Lärmpegelmessinstrument“, „Medikamente“, „Nachschlagewerk“, „Operationslehre“, „Plastische Chirurgie“, „Rauchentwöhnung“, „Schlafmedizin“, „Stimme“ und „Tinnitus“. Die Apps wurden mit Hilfe eines standardisierten Bewertungsmaßstabes, der sogenannten „App Synopsis für Nutzer“, evaluiert, mit dem sich zuverlässig einschätzen lässt, wie sicher eine solche Anwendung in der Patientenversorgung einzusetzen ist. Diese Checkliste enthält insgesamt 39 Fragen, die mit „ja“, „nein“ oder „unklar“ beantwortet werden können. Die Fragen sind den Kategorien „Medizinprodukt“, „Zweckbeschreibung“, „Funktionalität“, „Wissenschaftlichkeit“, „Einschränkungen und Limits“, „Risiken“, „Verlässlichkeit und Inhalte“, „Datenschutz“ und „Impressum“ zugeordnet. Die Antworten zu einigen Fragen sind in den Ampelfarben markiert, so dass der Nutzer schon beim Ankreuzen das Risiko des Einsatzes der App einfacher beurteilen kann (rot=hohes Risiko/ grün = niedriges Risiko). Insgesamt ergab die Evaluation mit diesem Bewertungstool, dass keine der Apps uneingeschränkt für den Einsatz empfohlen werden konnte. Insbesondere zeigte sich, dass die Möglichkeiten, Apps zu zertifizieren (Medizinprodukt oder CE-Kennzeichnung), kaum eingesetzt werden und häufig die Angaben zu dem aktuellen Stand von Wissenschaft und Technik fehlen. Aber auch eine fehlende Transparenz im Hinblick auf Einschränkungen und Limitationen wie auch datenschutzrechtliche und von der Nutzung der App ausgehende Risiken wurden moniert. Der Einsatz dieses strukturierten Bewertungsmaßstabes erlaubte eine präzise und übersichtliche Evaluation der Apps, insbesondere mit einem geringen zeitlichen Aufwand. Somit steht dem Nutzer ein Hilfsmittel zur Verfügung, mit dem Nutzen und Risiko von medizinischen Apps einfach abgewogen werden können.

Nach der Analyse der auf dem Markt befindlichen Apps mit Bezug zur HNO-Heilkunde entstand das Ziel, selbst eine in Bezug auf Funktionalität und Verlässlichkeit der Inhalte ausgewogene und transparente App zu entwickeln. Dabei sollten alle rechtlichen Vorgaben in Bezug auf die Datenverarbeitung und -speicherung erfüllt werden und reelle Chancen für eine Zertifizierung als Medizinprodukt enthalten sein. Nach ausgiebiger Recherche wurde entschieden, eine App zum Training der mimischen Muskulatur bei Fazialisparese zu entwickeln, da diesbezüglich ein offensichtlicher Bedarf evident wurde. Es ist bekannt, dass gezielte motorische Übungen zur Reanimation einer gelähmten mimischen Muskulatur im Sinne eines neuromuskulären Trainings zu messbaren Fortschritten führen können und darüber hinaus Unterstützung leisten, Syn- und Hyperkinesien zu vermeiden. Dieses bewährte und wissenschaftliche evaluierte Rehabilitationskonzept bietet sich demnach auch für eine mobile Anwendung an. Auf dem derzeitigen App-Markt gibt es keine vergleichbare Anwendung, lediglich Übungsbögen in digitaler Form sind vorhanden. Als Grundfunktionen der App wurden für Patienten verständliche Erklärungen über Anatomie und (Patho-) Physiologie des Nervus facialis, ein Trainingsmodus mit Anleitungen zu mimischen Übungen mit visuellem Feedback über die Frontkamera und eine Erinnerungsfunktion definiert. Zusätzlich sollte die App mit entsprechendem Hinweisen zu Haftungsbestimmungen und zum Datenschutz gemäß der neuen Datenschutz-Grundverordnung verse- 
hen werden. Die Konzeptionierung und die gewählte Vorgehensweise für die neue App zeigten, dass es möglich ist, eine Anwendungssoftware zu entwickeln, die das Potential hat, die Kriterien einer guten und qualitativ hochwertigen App zu erfüllen. Die weitere Validierung der App wird zeigen, ob die Funktionen korrekt implementiert sind und darüber hinaus, ob diese einen Benefit für den Patienten hat. Diese Erkenntnisse werden die Grundlage für den weiteren Entwicklungsprozess, aber auch für eine mögliche Zertifizierung der App als Medizinprodukt sein.

Im Rahmen des Referates konnte aufgezeigt werden, dass medizinische Apps trotz aller Risiken immer mehr an Bedeutung gewinnen. Dies trifft auch für den Bereich der HNO-Heilkunde zu, da hier für fast alle Teilbereiche des Faches schon Apps zur Verfügung stehen. Dies umfasst den Bereich der Diagnostik, z. B. App basierte audiometrischen Untersuchungen, bildgebende Diagnostik mittels App Kamera oder Erstellung eines Stimmprofiles. Aber auch therapeutisch können Apps eingesetzt werden, z. B. beim Tinntius Retraining, in der Schlafmedizin oder zur Modulation von Hörgeräteeinstellungen. Zusätzlich sind Apps auf dem Markt, die zum Bereich der Informationsgeber gezählt werden können, wie z. B. Nachschlagewerke für HNO-Erkrankungen, digitale Operationslehren oder Vorhersagen des Pollenfluges. Apps können auch zur Dokumentation eingesetzt werden, z. B. bei der Erhebung von allergischen Symptomen über eine visuelle Analog-Skala oder bei der Erfassung Tumor-spezifischer Symptome. Dies zeigt, dass der Einsatz solcher Apps das gesamte Fachgebiet der HNO-Heilkunde umfassen kann, wobei entscheidende Kriterien zur Qualität und Nachhaltigkeit auf wissenschaftlicher Basis noch erarbeitet werden müssen.

Mobile Health bietet somit ein großes Potenzial für das Fach der HNO-Heilkunde. Die flächendeckende Verfügbarkeit der Technik gepaart mit einem Gründergeist für praxis-orientierte Anwendungen bieten unserem Gesundheitssystem viele Chancen für neue Lösungsansätze. Basis dafür muss die Entwicklung hochwertiger, zuverlässiger und nachhaltiger Produkte sein, die einem noch zu definierenden Standard entsprechen müssen. Um dieses zu erreichen, ist die aktive Partizipation aller Akteure im Gesundheitswesen notwendig. Eine Möglichkeit, die Qualität von medizinischen Apps zu verbessern, sind Fächer und Berufsgruppen übergreifende Qualitätskriterien in Bezug auf ethische Unbedenklichkeit, inhaltliche Validität, Risikoangemessenheit, Transparenz und Zweckmäßigkeit sowie technische Faktoren. Diese gemeinschaftlich festzulegenden Kriterien zur Identifikation geeigneter Apps und deren Evaluation sollten an alle Beteiligten kommuniziert werden. Erst dadurch ist die erforderliche Transparenz zu erreichen, die Grundlage für eine allgemein anerkannte Nutzung solcher medizinischen Apps auch im Bereich der HNO-Heilkunde ist. Durch eine gezielte Qualitätssicherung können alle Seiten, Entwickler, Nutzer und Ärzteschaft, nachhaltig profitieren. Digitalisierung in der HNO-Heilkunde umfasst nicht nur den Bereich der medizinischen Apps, die Bedeutung des Themas spiegelt sich auch im Motto der 90. Jahresversammlung wider. Die Gründung der Kommission „Digitale Zukunft im Gesundheitswesen“ ist auch ein Baustein, um mobile Informations- und Kommunikationstechnologien im Kontext medizinscher und gesundheitsnaher Dienstleistungen gewinnbringend einzusetzen. Wir haben eine spannende Zukunft vor uns.
Interessenkonflikt

Die Autoren geben an, dass kein Interessenkonflikt besteht.

Literatur

[1] https://www.bitkom.org

[2] Albrecht U-V, Hillebrand U, von Jan U. Relevance of Trust Marks and CE Labels in German-Language Store Descriptions of Health Apps: Analysis. JMIR mHealth and uHealth 2018; 6: e10394

[3] Deutscher Ärzteverlag GmbH RDÄ. Internisten sichten und klassifizieren Gesundheits-Apps. 2018

[4] Albrecht U-V, Kuhn B, Land J et al. Nutzenbewertung von digitalen Gesundheitsprodukten (Digital Health) im gesellschaftlichen Erstattungskontext. Bundesgesundheitsblatt Gesundheitsforschung Gesundheitsschutz 2018; 61: 340-348

[5] Jee H. Review of researches on smartphone applications for physical activity promotion in healthy adults. J Exerc Rehabil 2017; 13: 3-11

[6] Middelweerd A, van der Laan DM, van Stralen MM et al. What features do Dutch university students prefer in a smartphone application for promotion of physical activity? A qualitative approach. Int J Behav Nutr Phys Act 2015; 12: 31

[7] Albrecht U-V. Chancen und Risiken von Gesundheits-Apps (CHARISMHA); engl. Chances and Risks of Mobile Health Apps (CHARISMHA). Hannover: Medizinische Hochschule Hannover; 2016

[8] [Anonym]. Medizinproduktegesetz in der Fassung der Bekanntmachung vom 7. August 2002 (BGBI. I S. 3146), das zuletzt durch Artikel 7 des Gesetzes vom 18. Juli 2017 (BGBI. I S. 2757) geändert worden ist. 1994; Im Internet: http://www.gesetze-im-internet.de/mpg/index. html

[9] Europäisches Parlament und R. Richtlinie 2007/47/EG des Europäischen Parlaments und des Rates. 2007

[10] Pramann O. Kapitel 10. Gesundheits-Apps und Datenschutz. In: Albrecht U-V, (Hrsg.). Chancen und Risiken von Gesundheits-Apps (CHARISMHA), engl Chances and Risks of Mobile Health Apps (CHARISMHA). Hannover: Medizinische Hochschule Hannover; 2016: 214-227

[11] Pramann O. Kapitel 11. Gesundheits-Apps als Medizinprodukte. In: Albrecht U-V, (Hrsg.). Chancen und Risiken von Gesundheits-Apps (CHARISMHA). Hannover: Medizinische Hochschule Hannover; 2016: 228-243

[12] Albrecht U-V, Pramann O. Haftungsfragen beim Einsatz von Gesundheits-Apps: Nobody is perfect. Dtsch Arztebl 2018; 115: A-520/B-452/C-452

[13] Hahn T. Tinnitus-Counseling: Neuer Selektivvertrag für HNO-Ärzte. HNO-Mitteilungen 2018; DOI: 166-167

[14] Wolf JA, Moreau JF, Akilov O et al. Diagnostic inaccuracy of smartphone applications for melanoma detection. JAMA Dermatol 2013; 149: 422-426

[15] Albrecht U-V. Gesundheits-Apps: Fachübergreifende Qualitätskriterien sind unabdingbar. Dtsch Arztebl 2018; 115: A-67/B-61/C-61

[16] Larrosa F, Rama-Lopez J, Benitez J et al. Development and evaluation of an audiology app for iPhone/iPad mobile devices. Acta Otolaryngol 2015; 135: 1119-1127

[17] Livshitz L, Ghanayim R, Kraus C et al. Application-Based Hearing Screening in the Elderly Population. Ann Otol Rhinol Laryngol 2017; 126: $36-41$

[18] Abu-Ghanem S, Handzel O, Ness L et al. Smartphone-based audiometric test for screening hearing loss in the elderly. Eur Arch Otorhinolaryngol 2016; 273: 333-339 
[19] Corry M, Sanders M, Searchfield GD. The accuracy and reliability of an app-based audiometer using consumer headphones: pure tone audiometry in a normal hearing group. International journal of audiology 2017; 56: 706-710

[20] Derin S, Cam OH, Beydilli $\mathrm{H}$ et al. Initial assessment of hearing loss using a mobile application for audiological evaluation. The Journal of laryngology and otology 2016; 130: 248-251

[21] Masalski M, Kipinski L, Grysinski T et al. Hearing Tests on Mobile Devices: Evaluation of the Reference Sound Level by Means of Biological Calibration. J Med Internet Res 2016; 18: e130

[22] Masalski M, Grysinski T, Krecicki T. Hearing Tests Based on Biologically Calibrated Mobile Devices: Comparison With Pure-Tone Audiometry. JMIR Mhealth Uhealth 2018; 6: e10

[23] Dewyer NA, jiradejvong P, Henderson Sabes J et al. Automated smartphone audiometry: Validation of a word recognition test app. Laryngoscope 2018; 128: 707-712

[24] [Anonym]. Hearing Check app. Nurs Stand 2016; 30: 31

[25] Sethi RKV, Ghanad I, Kanumuri V et al. Mobile Hearing Testing Applications and the Diagnosis of Sudden Sensorineural Hearing Loss: A Cautionary Tale. Otol Neurotol 2018; 39: e1-e4

[26] Saliba J, Al-Reefi M, Carriere JS et al. Accuracy of Mobile-Based Audiometry in the Evaluation of Hearing Loss in Quiet and Noisy Environments. Otolaryngol Head Neck Surg 2017; 156: 706-711

[27] Bright T, Pallawela D. Validated Smartphone-Based Apps for Ear and Hearing Assessments: A Review. JMIR Rehabil Assist Technol 2016; 3: e13

[28] Zamora W, Calafate CT, Cano JC. et al. Accurate Ambient Noise Assessment Using Smartphones. Sensors (Basel) 2017; 17:

[29] Kardous CA, Shaw PB. Evaluation of smartphone sound measurement applications. The Journal of the Acoustical Society of America 2014; 135: EL186-EL192

[30] Kardous CA, Shaw PB. Evaluation of smartphone sound measurement applications (apps) using external microphones-A follow-up study. The Journal of the Acoustical Society of America 2016; 140: EL327

[31] Neitzel RL, Heikkinen MS, Williams CC et al. Pilot study of methods and equipment for in-home noise level measurements. Appl Acoust 2015; 102: $1-11$

[32] Roberts B, Neitzel RL. Using Smart Devices to Measure Intermittent Noise in the Workplace. Noise \& health 2017; 19: 58-64

[33] Golub JS, Sharma A, Samy RN. A smartphone-optimized web site for conveniently viewing otolaryngology journal abstracts. Otolaryngol Head Neck Surg 2014; 151: 972-975

[34] Hsueh WD, Bent JP, Moskowitz HS. An app to enhance resident education in otolaryngology. Laryngoscope 2017, DOI: 10.1002/ lary. 27040

[35] Khan KM, Evans SS, Bielko SL et al. Efficacy of technology-based interventions to increase the use of hearing protections among adolescent farmworkers. International journal of audiology 2018; 57: 124-134

[36] Paglialonga A, Tognola G, Pinciroli F. Apps for Hearing Science and Care. Am J Audiol 2015; 24: 293-298

[37] Samra S, Wu A, Redleaf M. Interactive iPhone/iPad App for Increased Tympanic Membrane Familiarity. Ann Otol Rhinol Laryngol 2016; 125 : 997-1000

[38] Henry JA, Thielman E, Zaugg T et al. Development and field testing of a smartphone "App" for tinnitus management. International journal of audiology 2017; 56: 784-792

[39] Noorain Alam M, Gupta M, Munjal S et al. Efficacy of TRT Using Noise Presentation from Mobile Phone. Indian J Otolaryngol Head Neck Surg 2017; 69: 333-337
[40] Wunderlich R, Stein A, Engell A et al. Evaluation of iPod-Based Automated Tinnitus Pitch Matching. Journal of the American Academy of Audiology 2015; 26: 205-212

[41] Schlee W, Pryss RC, Probst T et al. Measuring the Moment-to-Moment Variability of Tinnitus: The TrackYourTinnitus Smart Phone App. Front Aging Neurosci 2016; 8: 294

[42] Probst T, Pryss RC, Langguth B et al. Outpatient Tinnitus Clinic, Self-Help Web Platform, or Mobile Application to Recruit Tinnitus Study Samples? Front Aging Neurosci 2017; 9: 113

[43] Hauptmann C, Wegener A, Poppe $\mathrm{H}$ et al. Validation of a Mobile Device for Acoustic Coordinated Reset Neuromodulation Tinnitus Therapy. Journal of the American Academy of Audiology 2016; 27: 720-731

[44] Mistry N, Coulson C, George A. endoscope-i: an innovation in mobile endoscopic technology transforming the delivery of patient care in otolaryngology. Expert Rev Med Devices 2017; 14: 913-918

[45] Wu C], Wu SY, Chen PC et al. An innovative smartphone-based otorhinoendoscope and its application in mobile health and teleotolaryngology. J Med Internet Res 2014; 16: e71

[46] Shah MU, Sohal M, Valdez TA et al. iPhone otoscopes: Currently available, but reliable for tele-otoscopy in the hands of parents? Int J Pediatr Otorhinolaryngol 2018; 106: 59-63

[47] Liu H, Akiki S, Barrowman NJ et al. Mobile Endoscopy vs Video Tower: A Prospective Comparison of Video Quality and Diagnostic Accuracy. Otolaryngol Head Neck Surg 2016; 155: 575-580

[48] Moshtaghi O, Sahyouni R, Haidar YM et al. Smartphone-Enabled Otoscopy in Neurotology/Otology. Otolaryngol Head Neck Surg 2017; 156: 554-558

[49] Stippig A, Hubers U, Emerich M. Apps in sleep medicine. Sleep Breath 2015; 19: 411-417

[50] Cho SW, Wee JH, Yoo S et al. Effect of Lifestyle Modification Using a Smartphone Application on Obesity With Obstructive Sleep Apnea: A Short-term, Randomized Controlled Study. Clinical and experimental otorhinolaryngology 2018, DOI: 10.21053/ceo.2017.01284

[51] Nakano H, Hirayama K, Sadamitsu Y et al. Monitoring sound to quantify snoring and sleep apnea severity using a smartphone: proof of concept. J Clin Sleep Med 2014; 10: 73-78

[52] Ong AA, Gillespie MB. Overview of smartphone applications for sleep analysis. World J Otorhinolaryngol Head Neck Surg 2016; 2: 45-49

[53] Lorenz CP, Williams AJ. Sleep apps: what role do they play in clinical medicine? Curr Opin Pulm Med 2017; 23: 512-516

[54] Camacho M, Robertson M, Abdullatif ] et al. Smartphone apps for snoring. The Journal of laryngology and otology 2015; 129: 974-979

[55] Baron KG, Duffecy J, Reid K et al. Technology-Assisted Behavioral Intervention to Extend Sleep Duration: Development and Design of the Sleep Bunny Mobile App. JMIR Ment Health 2018; 5: e3

[56] Siau RTK, Goswamy J, Jones $S$ et al. Is OperaVOX a clinically useful tool for the assessment of voice in a general ENT clinic? BMC Ear Nose Throat Disord 2017; 17: 4

[57] Grillo EU. An Online Telepractice Model for the Prevention of Voice Disorders in Vocally Healthy Student Teachers Evaluated by a Smartphone Application. Perspect ASHA Spec Interest Groups 2017; 2: 63-78

[58] Mat Baki M, Wood G, Alston M et al. Reliability of OperaVOX against Multidimensional Voice Program (MDVP). Clin Otolaryngol 2015; 40: 22-28

[59] Manfredi C, Lebacq J, Cantarella G et al. Smartphones Offer New Opportunities in Clinical Voice Research. J Voice 2017; 31: 111 e111-111 e117 
[60] Brodsky JR, Cusick BA, Kawai K et al. Peripheral vestibular loss detected in pediatric patients using a smartphone-based test of the subjective visual vertical. Int J Pediatr Otorhinolaryngol 2015; 79: 2094-2098

[61] Organ B, Liu H, Bromwich M. An iPhone-assisted particle repositioning maneuver for benign paroxysmal positional vertigo (BPPV): a prospective randomized study. J Am Board Fam Med 2015; 28 : $118-120$

[62] Whittaker M, Mathew A, Kanani R et al. Assessing the Unterberger test: introduction of a novel smartphone application. The Journal of laryngology and otology 2014; 128: 958-960

[63] Constantinescu G, Loewen I, King B et al. Designing a Mobile Health App for Patients With Dysphagia Following Head and Neck Cancer: A Qualitative Study. JMIR Rehabil Assist Technol 2017; 4: e3

[64] Starmer HM, Abrams R, Webster K et al. Feasibility of a Mobile Application to Enhance Swallowing Therapy for Patients Undergoing Radiation-Based Treatment for Head and Neck Cancer. Dysphagia 2018; 33: 227-233

[65] Lopez EA, Costa OA, Ferrari DV. Development and Technical Validation of the Mobile Based Assistive Listening System: A Smartphone-Based Remote Microphone. Am J Audiol 2016; 25: 288-294

[66] Maidment DW, Barker AB, Xia J et al. Effectiveness of alternative listening devices to conventional hearing aids for adults with hearing loss: a systematic review protocol. BMJ Open 2016; 6: e011683

[67] Ng SL, Phelan S, Leonard M et al. A Qualitative Case Study of Smartphone-Connected Hearing Aids: Influences on Patients, Clinicians, and Patient-Clinician Interactions. Journal of the American Academy of Audiology 2017; 28: 506-521

[68] Panahi I, Kehtarnavaz N, Thibodeau L. Smartphone-based noise adaptive speech enhancement for hearing aid applications. Conference proceedings : Annual International Conference of the IEEE Engineering in Medicine and Biology Society IEEE Engineering in Medicine and Biology Society Annual Conference 2016; 2016: 85-88

[69] Reddy CK, Yiya H, Panahi I. Two microphones spectral-coherence based speech enhancement for hearing aids using smartphone as an assistive device. Conference proceedings : Annual International Conference of the IEEE Engineering in Medicine and Biology Society IEEE Engineering in Medicine and Biology Society Annual Conference 2016; 2016: 3670-3673

[70] Reddy CKA, Shankar N, Bhat GS et al. An individualized super-Gaussian single microphone Speech Enhancement for hearing aid users with smartphone as an assistive device. IEEE Signal Process Lett 2017; 24: 1601-1605

[71] Yu R, Yiya H, Panahi IM et al. Smartphone-based real-time speech enhancement for improving hearing aids speech perception. Conference proceedings : Annual International Conference of the IEEE Engineering in Medicine and Biology Society IEEE Engineering in Medicine and Biology Society Annual Conference 2016; 2016 : 5885-5888

[72] Kessel KA, Vogel MM, Kessel C et al. Mobile Health in Oncology: A Patient Survey About App-Assisted Cancer Care. JMIR Mhealth Uhealth 2017; 5: e81

[73] Gomes MS, Bonan PR, Ferreira VY et al. Development of a mobile application for oral cancer screening. Technol Health Care 2017; 25: 187-195

[74] Duman-Lubberding S, van Uden-Kraan CF, Jansen F et al. Feasibility of an eHealth application "OncoKompas" to improve personalized survivorship cancer care. Support Care Cancer 2016; 24: 2163-2171
[75] Klimek L, Bergmann KC, Biedermann T et al. Visual analogue scales (VAS): Measuring instruments for the documentation of symptoms and therapy monitoring in cases of allergic rhinitis in everyday health care: Position Paper of the German Society of Allergology (AeDA) and the German Society of Allergy and Clinical Immunology (DGAKI), ENT Section, in collaboration with the working group on Clinical Immunology, Allergology and Environmental Medicine of the German Society of Otorhinolaryngology, Head and Neck Surgery (DGHNOKHC). Allergo J Int 2017; 26: 16-24

[76] Caimmi D, Baiz N, Tanno LK et al. Validation of the MASK-rhinitis visual analogue scale on smartphone screens to assess allergic rhinitis control. Clin Exp Allergy 2017; 47: 1526-1533

[77] Bousquet J, Devillier P, Arnavielhe $S$ et al. Treatment of allergic rhinitis using mobile technology with real-world data: The MASK observational pilot study. Allergy 2018, DOI: 10.1111/all.13406

[78] Bousquet J, Bewick M, Arnavielhe S et al. Work productivity in rhinitis using cell phones: The MASK pilot study. Allergy 2017; 72: 1475-1484

[79] Bourret R, Bousquet J, Mercier J et al. MASK-rhinitis, a single tool for integrated care pathways in allergic rhinitis. World Hosp Health Serv 2015; 51: 36-39

[80] Bianchi A, Tsilochristou O, Gabrielli F et al. The Smartphone: A Novel Diagnostic Tool in Pollen Allergy? J Investig Allergol Clin Immunol 2016; 26: 204-207

[81] Fiedler L, Obleser J, Lunner T et al. Ear-EEG allows extraction of neural responses in challenging listening scenarios - A future technology for hearing aids? Conference proceedings: Annual International Conference of the IEEE Engineering in Medicine and Biology Society IEEE Engineering in Medicine and Biology Society Annual Conference 2016; 2016: 5697-5700

[82] von Rosenberg W, Chanwimalueang T, Goverdovsky V et al. Hearables: feasibility of recording cardiac rhythms from head and in-ear locations. R Soc Open Sci 2017; 4: 171214

[83] Goverdovsky V, von Rosenberg W, Nakamura T et al. Hearables: Multimodal physiological in-ear sensing. Sci Rep 2017; 7: 6948

[84] Albrecht UV, Jungnicke T, von Jan U. iSignIT - Communication App and Concept for the Deaf and Hard of Hearing. Stud Health Technol Inform 2015; 213: 283-286

[85] Bobian M, Kandinov A, El-Kashlan $\mathrm{N}$ et al. Mobile applications and patient education: Are currently available GERD mobile apps sufficient? Laryngoscope 2017; 127: 1775-1779

[86] Jethanamest D, Azadpour M, Zeman AM et al. A Smartphone Application for Customized Frequency Table Selection in Cochlear Implants. Otol Neurotol 2017; 38: e253-e261

[87] Larrosa F, Dura M], Roura J et al. Rhinoplasty planning with an iPhone app: analysis of otolaryngologists response. Eur Arch Otorhinolaryngol 2013; 270: 2473-2477

[88] Albrecht UV, von Jan U, Jungnickel T et al. App-synopsis - standard reporting for medical apps. Stud Health Technol Inform 2013; 192: 1154

[89] http://www.plrimedapplab.de

[90] Albrecht UV, Pramann O, von Jan U. App-Synopsis: Checkliste zur Selbsteinschätzung der Vertrauenswürdigkeit von Health-Apps. 2014, DOI: 341

[91] Baugh RF, Basura G], Ishii LE et al. Clinical practice guideline: Bell's palsy. Otolaryngol Head Neck Surg 2013; 149: S1-S27

[92] Butler DP, Grobbelaar AO. Facial palsy: what can the multidisciplinary team do? J Multidiscip Healthc 2017; 10: 377-381 
[93] Lindsay RW, Robinson M, Hadlock TA. Comprehensive facial rehabilitation improves function in people with facial paralysis: a 5-year experience at the Massachusetts Eye and Ear Infirmary. Phys Ther 2010; 90: 391-397

[94] Teixeira LJ, Soares BG, Vieira VP et al. Physical therapy for Bell s palsy (idiopathic facial paralysis). Cochrane Database Syst Rev 2008; DOI: 10.1002/14651858.CD006283.pub2 CD006283
[95] http://bellspalsytraining.com

[96] Pourmomeny AA, Zadmehr H, Hossaini M. Measurement of facial movements with Photoshop software during treatment of facial nerve palsy. J Res Med Sci 2011; 16: 1313-1318

[97] https://www.apple.com/de/researchkit/ 